\title{
돌 Fermilab
}

\section{FERMILAB- PUB-16-215-APC} ACCEPTED

June 2016

\section{Study of secondary neutron interactions with ${ }^{232} \mathrm{Th},{ }^{129} \mathrm{I}$, and ${ }^{127} \mathrm{I}$ nuclei with the uranium assembly "QUINTA " at 2, 4, and $8 \mathrm{GeV}$ deuteron beams of the JINR Nuclotron accelerator ${ }^{12}$}

\author{
J. Adam ${ }^{1,2}$, V.V. Chilap ${ }^{3}$, V.I. Furman ${ }^{1}$, M.G. Kadykov1, J. Khushvaktov ${ }^{1}$, V.S. Pronskikh ${ }^{1,4}$, \\ A.A. Solnyshkin ${ }^{1}$, V.I. Stegailov ${ }^{1}$, M. Suchopar ${ }^{2}$, V.M. Tsoupko-Sitnikov ${ }^{1}$, S.I. Tyutyunnikov ${ }^{1}$, \\ J. Vrzalova ${ }^{1}$, V. Wagner ${ }^{2}$, L. Zavorka ${ }^{1}$
}

${ }^{1}$ Joint Institute for Nuclear Research, Dubna, Russia.

${ }^{2}$ Nuclear Physics Institute ASCR PRI, Czech Republic.

${ }^{3}$ Center of Physical and Technical Projects “Atomenergomash”, Moscow, Russia.

${ }^{4}$ Fermi National Accelerator Laboratory, Batavia IL, USA

\begin{abstract}
The natural uranium assembly, "QUINTA", was irradiated with 2, 4, and $8 \mathrm{GeV}$ deuterons. The $232 \mathrm{Th}, 127 \mathrm{I}$, and 129 I samples have been exposed to secondary neutrons produced in the assembly at a $20-\mathrm{cm}$ radial distance from the deuteron beam axis. The spectra of gamma rays emitted by the activated $232 \mathrm{Th}, 127 \mathrm{I}$, and $129 \mathrm{I}$ samples have been analyzed and several tens of product nuclei have been identified. For each of those products, neutron-induced reaction rates have been determined. The transmutation power for the 129 I samples is estimated. Experimental results were compared to those calculated with well-known stochastic and deterministic codes.
\end{abstract}

\footnotetext{
${ }^{1}$ Work supported by Fermi Research Alliance, LLC under contract No. DE-AC02-07CH11359 with the U.S. Department of Energy. ${ }^{2}$ Accepted to Applied Radiation and Isotopes, 2015.
} 


\title{
Study of secondary neutron interactions with ${ }^{232} \mathrm{Th},{ }^{129} \mathrm{I}$, and ${ }^{127} \mathrm{I}$ nuclei with the uranium assembly " QUINTA " at 2, 4, and $8 \mathrm{GeV}$ deuteron beams of the JINR Nuclotron accelerator
}

\author{
J. Adam ${ }^{1,2}$, V.V. Chilap ${ }^{3}$, V.I. Furman ${ }^{1}$, M.G. Kadykov 1 , J. Khushvaktov ${ }^{1}$, V.S. Pronskikh ${ }^{1,4}$, \\ A.A. Solnyshkin ${ }^{1}$, V.I. Stegailov ${ }^{1}$, M. Suchopar ${ }^{2}$, V.M. Tsoupko-Sitnikov ${ }^{1}$, \\ S.I. Tyutyunnikov ${ }^{1}$, J. Vrzalova ${ }^{1}$, V. Wagner ${ }^{2}$, L. Zavorka ${ }^{1}$,
}

\author{
${ }^{1}$ Joint Institute for Nuclear Research, Dubna, Russia. \\ ${ }^{2}$ Nuclear Physics Institute ASCR PRI, Czech Republic. \\ ${ }^{3}$ Center of Physical and Technical Projects “Atomenergomash”, Moscow, Russia. \\ ${ }^{4}$ Fermi National Accelerator Laboratory, Batavia IL, USA
}

\section{INTRODUCTION}

Interest in the international scientific community for research of this kind, is primarily concerned with the problem of transmutation of long-lived radioactive waste $[1,2]$ and the creation of subcritical nuclear power plants with uraniumthorium cycle, controlled high-energy particle accelerators (Accelerator Driven Subcritical Systems) [3,4]. Such research is actively conducted throughout the world has been for the last two decades: PNF (Poahng) [5], n-ToF (CERN) [6], MYRRHA (Belgium) [7] and «Energy + Transmutation» setup at JINR (Dubna) [8, 9, 10, 11]. Currently working in this direction and develops a number of programs: SINQ (PSI) [12], KEK (Japan) [13], MYRRHA (Belgium), n-ToF (CERN) and a cluster of other research programs at LANL (USA) [14] - for obtaining data and developing new materials to create prototypes industrial ADS-systems.

During the past several years, such studies have been conducted and are ongoing with beams of particles Nuclotron VBLHEP JINR (Dubna) under the program Energy plus Transmutation of Radioactive Waste. This program was carried out a large number of experiments on subcritical uranium-lead target "QUINTA" [15, 16], as well as lead-graphite target "GAMMA-3" [17]. Several experiments were carried out using a solid lead target "GENERATOR" [18, 19, 20] with proton beam Phasotron DLNP JINR.

In this paper we present experimental data in comparison with the calculations obtained in the last two years in studying the interaction of secondary neutrons with 
nucleus ${ }^{232} \mathrm{Th},{ }^{129} \mathrm{I},{ }^{127} \mathrm{I}$ on the "QUINTA" VBLHEP JINR on deuteron beams with energies $2,4,8 \mathrm{GeV}$.

\section{STRUCTURE SETUP "QUINTA"}

Uranium assembly "Quinta" is presented in Fig.1. It consists of five sections, formed in the shape of a hexahedron (aluminum containers with an inscribed diameter of $284 \mathrm{~mm}$ ). Containers filled cylindrical rods of natural uranium metal, having a sealed aluminum shell (external dimensions: diameter $3.6 \mathrm{~cm}$, length 10.4 $\mathrm{cm}$, weight $1.72 \mathrm{~kg}$ of uranium). The ends are made of aluminum sections, $6 \mathrm{~mm}$ thick. The first section, the first located along the beam contains 54 uranium rod and has a through central opening $80 \mathrm{~mm}$ in diameter for the input beam into the target, made in order to reduce its albedo and reduce the leakage of neutrons from the target. Four subsequent sections are structurally identical and contain 61 uranium rods. Mass of uranium in one section is $61 \times 1.72=104.92 \mathrm{~kg}$, and the total mass of uranium entire target $298 \times 1.72=512.56 \mathrm{~kg}$. The fill factor of uranium $2,3,4$ or 5 sections about 0.8 , and the whole assembly of uranium $\sim 0.6$.

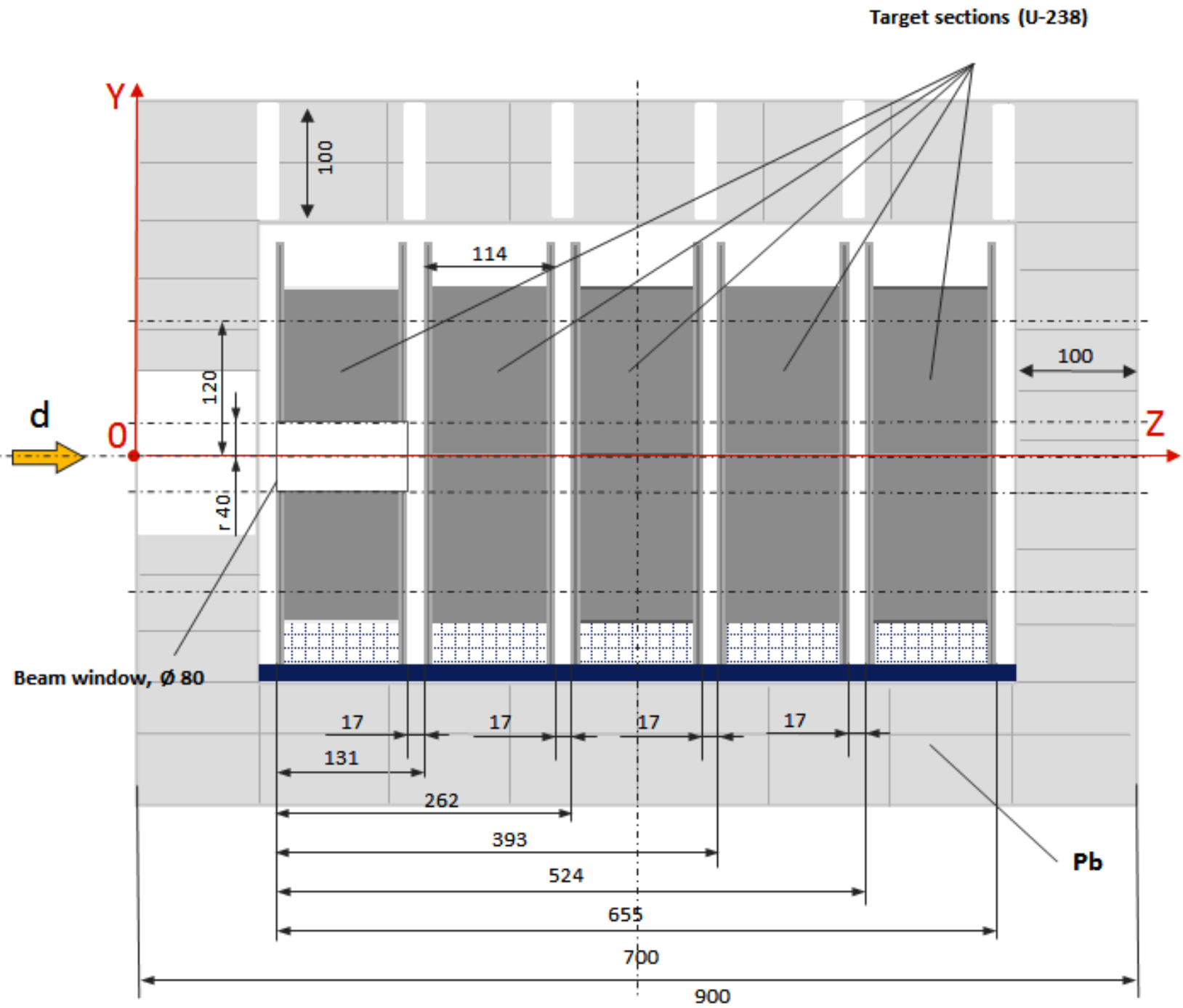


Uranium target surrounded with lead shield thickness of $10 \mathrm{~cm}$ and a weight of $2545 \mathrm{~kg}$ with a window to enter the beam dimensions of $15 \times 15 \mathrm{~cm}^{2}$ (see Fig. 2). In the side wall of lead shield on opposite to the third section there is window hole in the size $15 \times 5 \mathrm{~cm}^{2}$ to accommodate transmutation samples. The upper part of the lead assembly is provided with a special hole for mounting and dismantling of detector probes and installation of the samples inside the uranium assemblies between sections.

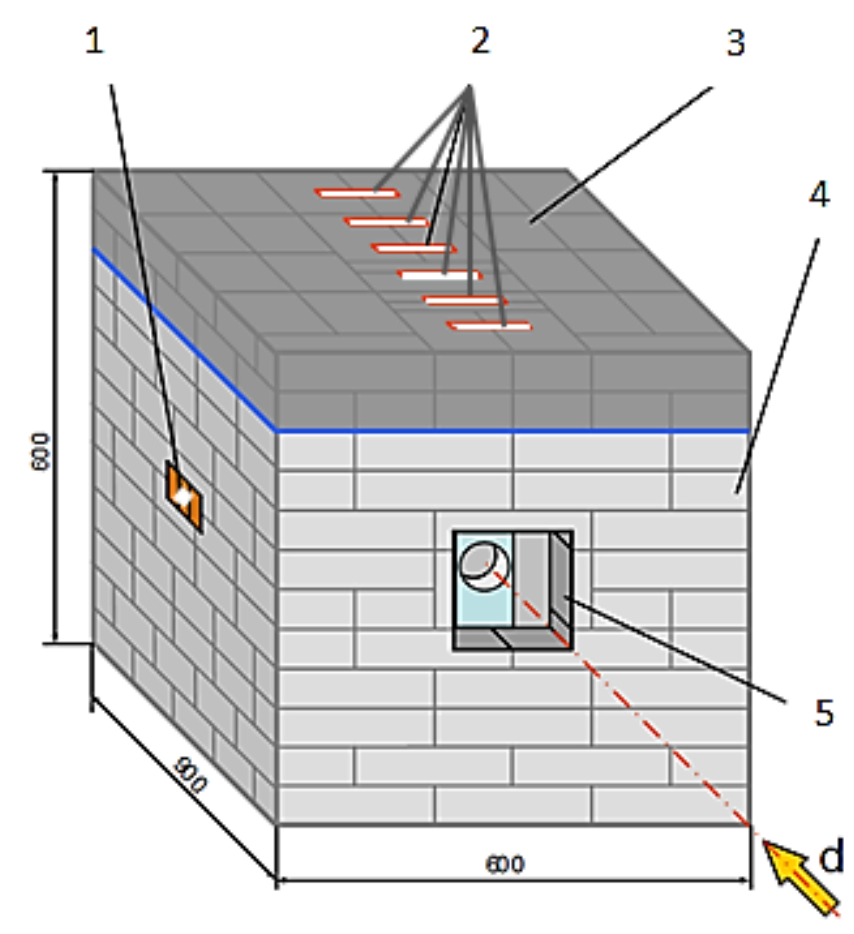

Fig. 2. The general view of setup "QUINTA", where 1 - window for placement of transmutation samples, 2 - mine for mounting and dismantling of detector probes and installation of the samples inside the uranium assemblies between sections, 3 - cover lead assembly, 4 - lead assembly, 5 input box beam $15 \times 15 \mathrm{~cm}^{2}$.

\section{EXPERIMENT}

In our experiments, transmutation samples $\left({ }^{127} \mathrm{I},{ }^{129} \mathrm{I},{ }^{\text {nat }} \mathrm{Th},{ }^{233} \mathrm{U},{ }^{235} \mathrm{U},{ }^{\text {nat }} \mathrm{U}\right.$, ${ }^{237} \mathrm{~Np},{ }^{238} \mathrm{Pu},{ }^{239} \mathrm{Pu}$, and ${ }^{241} \mathrm{Am}$ ) were placed inside the window 1 (see Fig. 2). Irradiation was carried out on three deuteron energies $(2,4$ and $8 \mathrm{GeV})$. Before entering the deuteron beam at the target were installed aluminum and copper foil. It is possible to determine the integral flux of deuterons and beam shape. Used the widely known method of activation of aluminum foil in the reaction ${ }^{27} \mathrm{Al}(\mathrm{d}, \mathrm{x})^{24} \mathrm{Na}$ and copper foil in the reaction ${ }^{n a t} \mathrm{Cu}(\mathrm{d}, \mathrm{x})^{24} \mathrm{Na}$. The following Table 1 shows the irradiation conditions and the characteristics of the samples that were used in our studies ( ${ }^{\text {nat }} \mathrm{Th},{ }^{129} \mathrm{I}$ and ${ }^{127} \mathrm{I}$ ).

Table.1. Data on the irradiation conditions and characteristics of the samples. 


\begin{tabular}{|c|c|c|c|c|c|c|}
\hline Deuterons energy, GeV & \multicolumn{2}{|c|}{2} & \multicolumn{2}{|c|}{4} & \multicolumn{2}{|c|}{8} \\
\hline Irradiation time, min. & \multicolumn{2}{|c|}{376} & \multicolumn{2}{|c|}{561} & \multicolumn{2}{|c|}{970} \\
\hline $\begin{array}{l}\text { Integral number of } \\
\text { deuterons }\end{array}$ & \multicolumn{2}{|c|}{$3.02(10) \mathrm{E}+13$} & \multicolumn{2}{|c|}{$2.73(10) \mathrm{E}+13$} & \multicolumn{2}{|c|}{$9.10(40) \mathrm{E}+12$} \\
\hline \multirow{2}{*}{$\begin{array}{l}\text { Coordinates of the center } \\
\text { of beam, } \mathrm{cm} *\end{array}$} & $\mathrm{Xc}$ & Yc & $\mathrm{Xc}$ & Yc & $\mathrm{Xc}$ & Yc \\
\hline & $1.5(2)$ & $0.1(1)$ & $1.8(1)$ & $-0.3(1)$ & $0.9(1)$ & $0.1(1)$ \\
\hline \multirow{2}{*}{$\begin{array}{l}\text { FWHM (full width at half } \\
\text { maximum), } \mathrm{cm} *\end{array}$} & FWHM $_{\mathrm{X}}$ & $\mathrm{FWHM}_{\mathrm{Y}}$ & FWHM $_{\mathrm{X}}$ & FWHM $_{Y}$ & FWHM $_{\mathrm{X}}$ & FWHM $_{Y}$ \\
\hline & $2.0(1)$ & $1.7(2)$ & $1.5(2)$ & $1.1(1)$ & $1.0(1)$ & $1.3(1)$ \\
\hline Samples & \multicolumn{2}{|c|}{ Th-nat } & \multicolumn{2}{|c|}{ Th-nat } & \multicolumn{2}{|c|}{ Th-nat } \\
\hline Mass, g. & \multicolumn{2}{|c|}{0.975} & \multicolumn{2}{|c|}{1.000} & \multicolumn{2}{|c|}{0.249} \\
\hline Diameter of samples, $\mathrm{cm}$ & \multicolumn{2}{|c|}{1.3} & \multicolumn{2}{|c|}{1.3} & \multicolumn{2}{|c|}{1.3} \\
\hline Samples & $\mathrm{I}-129$ & $\mathrm{I}-127$ & $\mathrm{I}-129$ & $\mathrm{I}-127$ & $\mathrm{I}-129$ & $\mathrm{I}-127$ \\
\hline Mass (I-129), g. & 0.591 & - & 0.339 & - & 0.218 & - \\
\hline Mass (I-127), g. & 0.129 & 1.550 & 0.074 & 1.270 & 0.048 & 1.980 \\
\hline Mass (Na-23), g. & 0.118 & 0.290 & 0.067 & 0.230 & 0.043 & 0.360 \\
\hline Mass (Al-27), g. ** & 17.6 & - & 17.6 & - & 17.6 & - \\
\hline Diameter of samples, $\mathrm{cm}$ & 2.1 & 2.0 & 2.1 & 2.0 & 2.1 & 2.0 \\
\hline
\end{tabular}

* Deuteron beam parameters (plata0) from group I.V. Zhuk (Minsk). Determined using solidstate nuclear track detectors with radiators from a natural lead, called sensors [private message].

${ }^{* *}$ Container for radioactive ${ }^{129} \mathrm{I}$.

After each session of irradiation the studying samples were transported to the DLNP by complex YASNAPP-2 where measured $\gamma$-spectra with the three spectrometers based HPGe-detector ORTEC (single detector efficiency - 33\% energy resolution - $1.8 \mathrm{keV}$ at line $1.33 \mathrm{MeV}{ }^{60} \mathrm{Co}$ ) and CANBERRA (two detector efficiency $-18 \%$ and $30 \%$, the energy resolutions $-1.9 \mathrm{keV}$ and $1.8 \mathrm{keV} 1.33 \mathrm{MeV}$ at line ${ }^{60} \mathrm{Co}$ ). For each sample at various time intervals was measured from 13 to 16 $\gamma$-spectra and decay time to the first spectrum from 79 to $157 \mathrm{~min}$. Calibration of the detectors by energy and efficiency was performed using a standard set of sources $\left({ }^{54} \mathrm{Mn},{ }^{57} \mathrm{Co},{ }^{60} \mathrm{Co},{ }^{88} \mathrm{Y},{ }^{113} \mathrm{Sn},{ }^{133} \mathrm{Ba},{ }^{137} \mathrm{Cs},{ }^{139} \mathrm{Ce},{ }^{152} \mathrm{Eu},{ }^{228} \mathrm{Th},{ }^{241} \mathrm{Am}\right)$.

\section{PROCESSING OF $\gamma$ - SPECTRA}

Processing of the measured $\gamma$-spectra were using DEIMOS32 [21]. The program allows to define the area under the $\gamma$-peaks and their position (channel number). Next, using a special software package [22] was calibrated for energy, 
corrects for detector efficiency and identified separate $\gamma$-lines corresponding nuclei products that were emitted in the sample as a result of interaction with secondary neutrons. In determining the intensities of $\gamma$-transitions also introduced corrections to the nuclear decay products during irradiation, corrections for self-absorption for registered $\gamma$-rays in the sample, to the geometric dimensions of the sample, corrections to breaks during irradiation and the change in intensity of the deuteron beam (on-line measurements of fast ionization chambers). All these procedures are described in detail in $[9,22,23]$.

\section{METHOD OF ANALYSIS AND CALCULATIONS}

In determining the reaction rates (experimental data) used the following relation (1) [9]:

$$
R\left(A_{r}, Z_{r}\right)=\frac{Q\left(A_{r}, Z_{r}\right)}{N_{t} N_{d}},
$$

where, $Q\left(A_{r}, Z_{r}\right)$ - rate of production of radioactive nucleus $(\mathrm{r}), N_{t}$ - number of atoms in the sample, $N_{d}$ - number of incident deuterons on the target.

Values of reaction rates (in calculation) calculated by the formula:

$R\left(A_{r}, Z_{r}\right)=\int_{E_{t h r}\left(A_{r}, Z_{r}\right)}^{\infty} \sigma\left(A_{r}, Z_{r}, E_{n}\right) \varphi\left(E_{n}\right) d E_{n}$

where, $\sigma\left(A_{r}, Z_{r}, E_{n}\right)$ - reaction cross section, $\varphi\left(E_{n}\right)$ - neutron fluence.

Calculations of reaction rates (Calc.1) were performed with program MARS15 [24] and the reaction products with neutrons were modeled using LAQGSM03.03 [25].

Calculation of neutron fluence (Calc.2) was carried with program MCNPX2.7 [26] using models INCL4 (intranuclear physics model) [27] and ABLA (fissionevaporation model) [28]. For the reaction $(\mathrm{n}, \gamma)$ in the ${ }^{232} \mathrm{Th}$, the reaction cross sections were calculated by the program TALYS1.4 [29], and (n,fission) were taken from the nuclear data library TENDL-2009 [30], as in the TENDL-2009, thorium fission reaction cross sections with neutrons calculated up to neutron energy 200 $\mathrm{MeV}$ and are in good agreement with the data from the library JEFF3.1.2 [31], as well as with the experimental data [32] (see Figure.3). Reaction cross sections for $(\mathrm{n}, \gamma),(\mathrm{n}, 4 \mathrm{n})$ and $(\mathrm{n}, 6 \mathrm{n})$ in ${ }^{129} \mathrm{I}$ to $40 \mathrm{MeV}$ from TENDL-2011, from 40 to $200 \mathrm{MeV}$, the calculation was performed with program TALYS1.4. In ${ }^{127}$ I reaction cross sections for $(n, \gamma),(n, 2 n)$ and $(n, 4 n)$ chosen the calculations with program TALYS1.4 lack of data in libraries up to $200 \mathrm{MeV}$. 


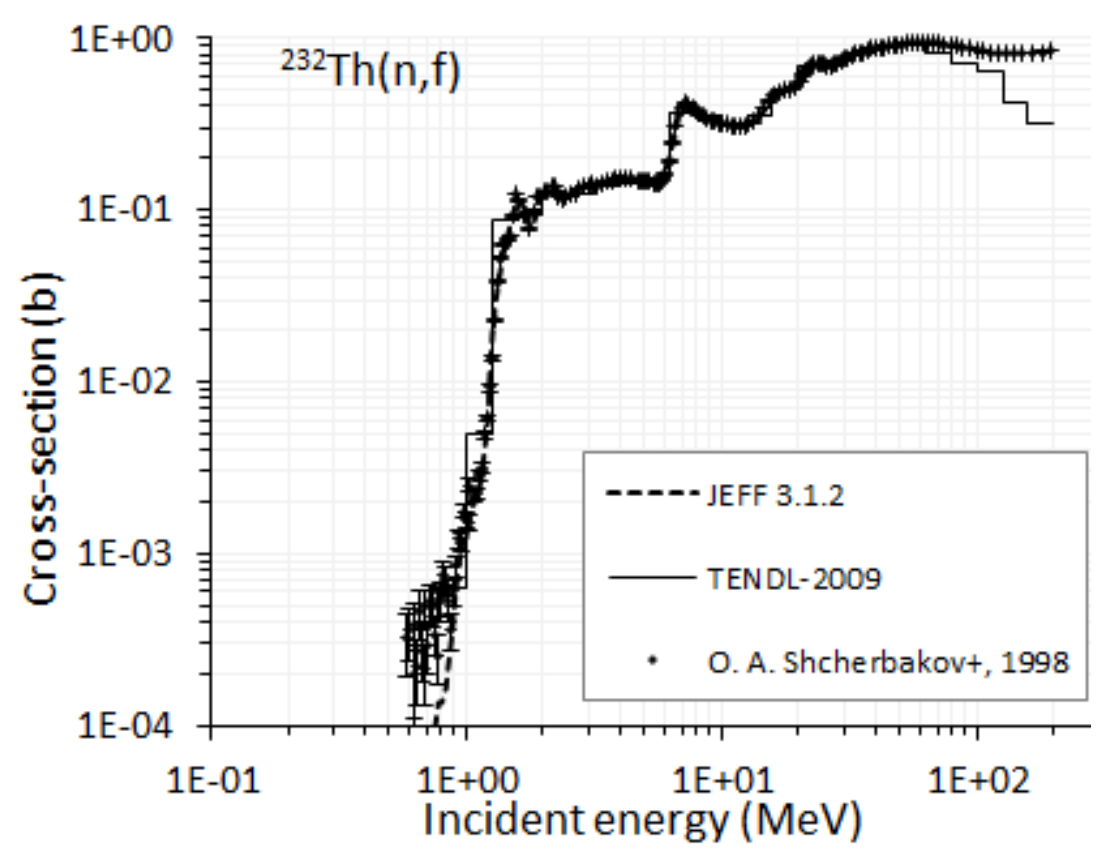

Fig.3. Comparison of the calculated fission cross sections of ${ }^{232} \mathrm{Th}$ with experiment [32], depending on the neutron energy.

\section{RESULTS OF ${ }^{232}$ Th}

In results processing of obtained data in the experiment for thorium has been identified a large number of product nuclei (at $2 \mathrm{GeV}-19$, at $4 \mathrm{GeV}-30$ and at 8 $\mathrm{GeV}$ - 27) for three values of the deuteron energy. For each of these are obtained reaction rates. Table 2 summarizes the results of ${ }^{232} \mathrm{Th}$ for all energies for all registered nuclei. $\mathrm{I}_{\mathrm{g}}$ - the yield of gamma rays (\%), $\mathrm{T}_{1 / 2}($ Exper $)$ - the observed halflives of the radionuclides, $\mathrm{R}$ - reaction rate, $\langle\mathrm{R}\rangle$ - the average value of the reaction rate $\left(\right.$ atoms $^{-1} *$ deuteron $\left.^{-1}\right)$. The results show that with increasing deuteron energy increases and the value of the reaction rates for almost all product nuclei. Obviously, this growth is due to the increased flow and energy of secondary neutrons with increasing energy deuterons. Fig. 4 shows the ratio of reaction rate $\mathrm{R}(4 \mathrm{GeV}) / \mathrm{R}(2$ $\mathrm{GeV})$ and $\mathrm{R}(8 \mathrm{GeV}) / \mathrm{R}(2 \mathrm{GeV})$ for the identified product nuclei generated in reactions with secondary neutrons at all three deuteron energies $(2,4,8 \mathrm{GeV})$. 


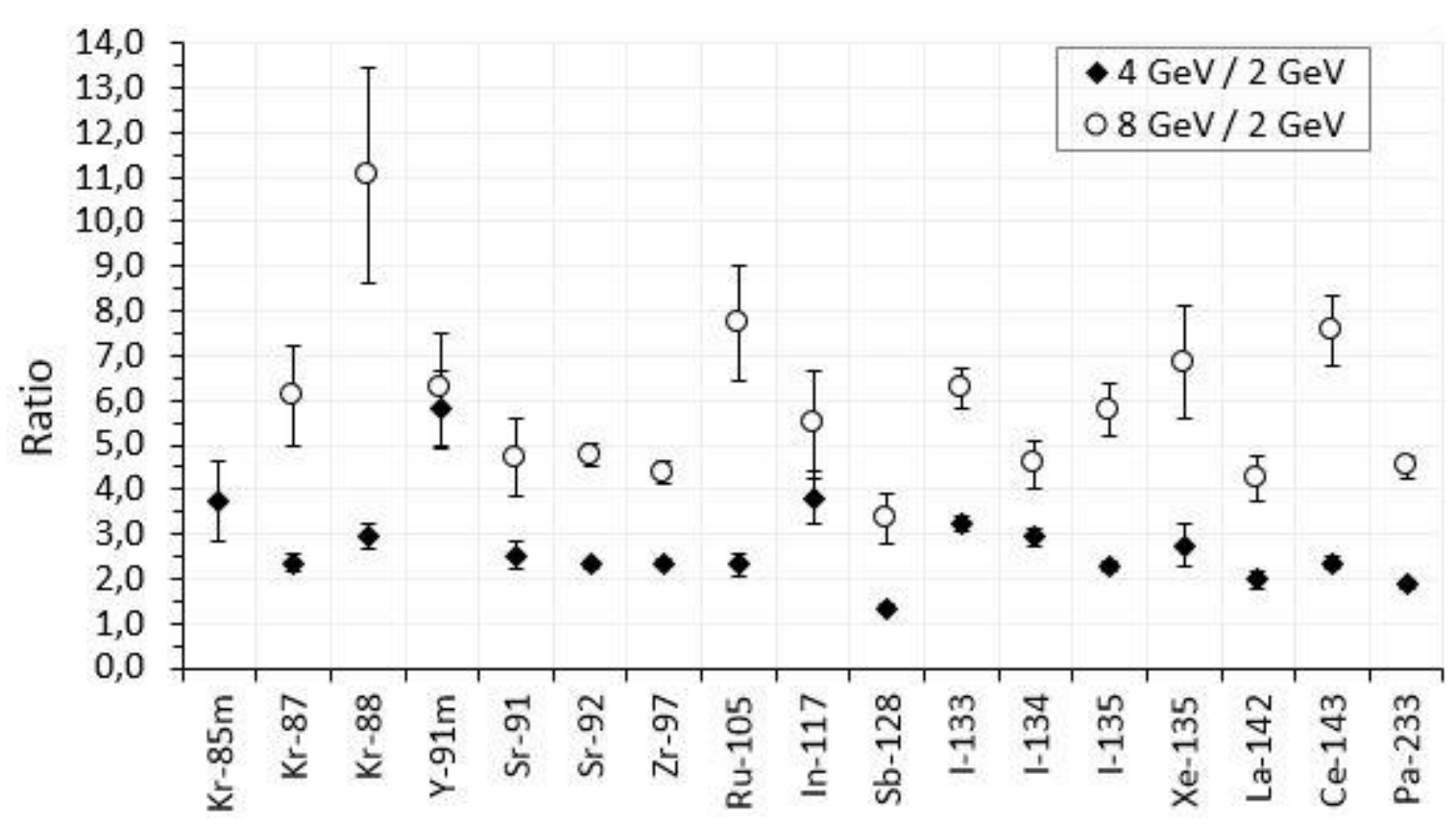

Fig.4. Experimental values of the ratio of reaction rate $\mathrm{R}(4 \mathrm{GeV}) / \mathrm{R}(2 \mathrm{GeV})$ and $\mathrm{R}(8 \mathrm{GeV}) / \mathrm{R}(2$ $\mathrm{GeV}$ ) for ${ }^{232} \mathrm{Th}$ with secondary neutrons for product nuclei at energies of deuterons $2,4,8 \mathrm{GeV}$.

Presented in Fig. 4 nuclei products: ${ }^{85 \mathrm{~m}} \mathrm{Kr},{ }^{87} \mathrm{Kr},{ }^{88} \mathrm{Kr},{ }^{91 \mathrm{~m}} \mathrm{Y},{ }^{91} \mathrm{Sr},{ }^{92} \mathrm{Y},{ }^{92} \mathrm{Sr},{ }^{93} \mathrm{Y}$, ${ }^{97} \mathrm{Nb},{ }^{97} \mathrm{Zr},{ }^{133} \mathrm{I},{ }^{134} \mathrm{I},{ }^{135} \mathrm{I},{ }^{135} \mathrm{Xe},{ }^{138} \mathrm{Cs},{ }^{142} \mathrm{La}$ and ${ }^{143} \mathrm{Ce}$ - produced by fission the ${ }^{232} \mathrm{Th}$; radionuclides: ${ }^{66} \mathrm{Ga},{ }^{88} \mathrm{Y},{ }^{92 \mathrm{~m}} \mathrm{Nb},{ }^{105} \mathrm{Ru},{ }^{115} \mathrm{Cd},{ }^{115 \mathrm{~m}} \mathrm{In},{ }^{117} \mathrm{In}{ }^{126} \mathrm{Sb},{ }^{128} \mathrm{Sb},{ }^{129} \mathrm{Sb},{ }^{132} \mathrm{Te}$ и ${ }^{132} \mathrm{I}$ - products of the (n,spallation) reactions (the ratio of the reaction rate to yield $\mathrm{R}$ / $\mathrm{Y}$ for these radionuclides $10-20$ times differed from fission products). ${ }^{224} \mathrm{Ac}$ is product of reaction ${ }^{228} \mathrm{Th}(\mathrm{n}, 2 \mathrm{nt}){ }^{224} \mathrm{Ac}\left(\mathrm{E}_{\mathrm{thr}}=16.56 \mathrm{MeV}\right),{ }^{233} \mathrm{~Pa}$ produced in the reaction ${ }^{232} \mathrm{Th}(\mathrm{n}, \gamma){ }^{233} \mathrm{Th}\left(\beta^{-}\right.$decay, $\left.\mathrm{T}_{1 / 2}=22.3 \mathrm{~min}.\right) \rightarrow{ }^{233} \mathrm{~Pa}\left(\beta^{-}\right.$decay, $\mathrm{T}_{1 / 2}=26.967$ day) $\rightarrow{ }^{233} \mathrm{U}$.

Fig. 5 shows the experimental values of the reaction rate $(n, \gamma)$ and fission $(n, f)$ depending on the deuteron energy in comparison with the calculations Calc. 2 (MCNPX). In both cases, there is a proportional increase in the values of reaction rate with increasing energy deuterons. 


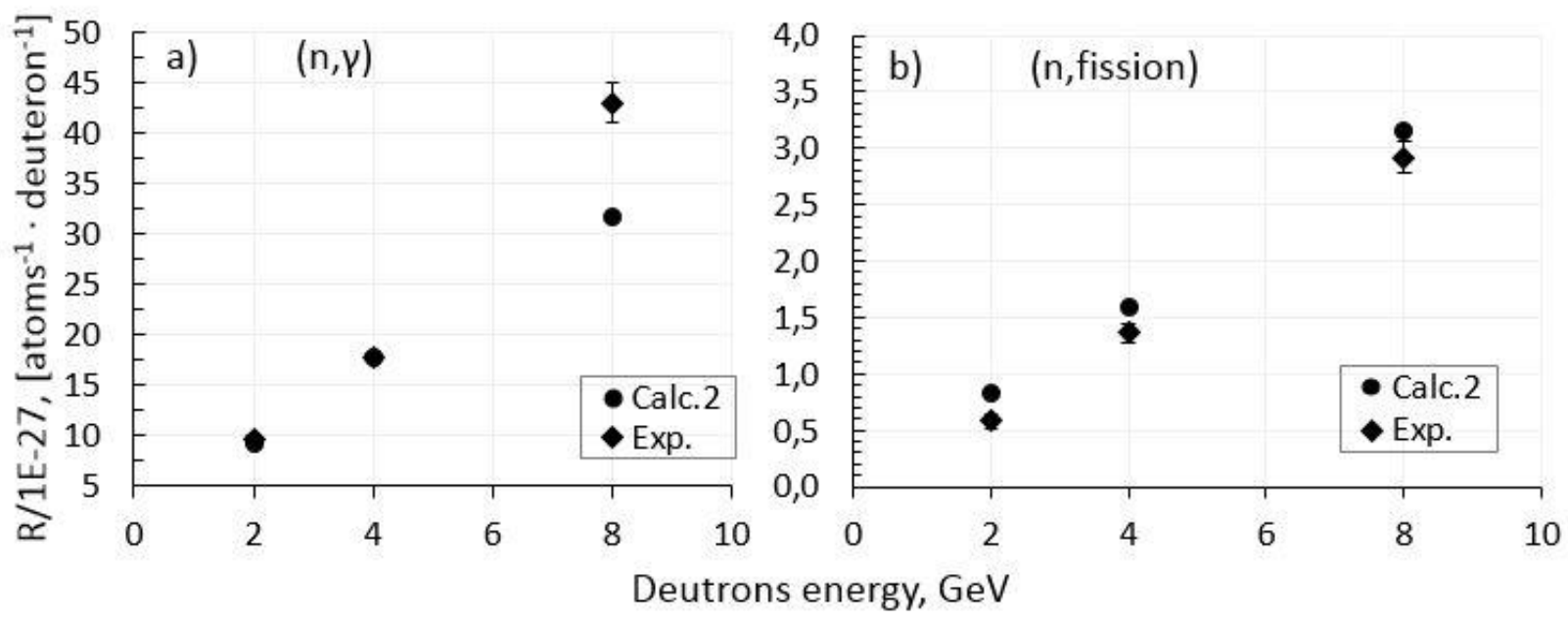

Fig.5. a) Comparison of experimental and calculated values of the reaction rate (n, $\gamma){ }^{232} \mathrm{Th}$ in the interaction with the secondary neutrons, depending from deuteron energy, b) Comparison of experimental and calculated values of the fission reaction rate $(n, f)$ in the interaction of ${ }^{232} \mathrm{Th}$ with secondary neutrons depending from deuteron energy.

Calculations of fission reaction rates $\mathrm{R}(\mathrm{n}, \mathrm{f})$ from the experimental data were carried out as follows. Cumulative yield $\mathrm{Y}$ for fission products of ${ }^{232} \mathrm{Th}$ were taken from the library JEFF3.1 for neutrons with an energy $0.4 \mathrm{MeV}$. The average value of the ratio R/Y for fission products such as nuclei ${ }^{85 \mathrm{~m}} \mathrm{Kr},{ }^{87} \mathrm{Kr},{ }^{88} \mathrm{Kr},{ }^{91 \mathrm{~m}} \mathrm{Y},{ }^{91} \mathrm{Sr},{ }^{92} \mathrm{Y}$, ${ }^{92} \mathrm{Sr},{ }^{93} \mathrm{Y},{ }^{97} \mathrm{Nb},{ }^{97} \mathrm{Zr},{ }^{133} \mathrm{I},{ }^{134} \mathrm{I},{ }^{135} \mathrm{I},{ }^{135} \mathrm{Xe},{ }^{138} \mathrm{Cs},{ }^{142} \mathrm{La}$ and ${ }^{143} \mathrm{Ce}$ in experiment for deuteron energies at $2 \mathrm{GeV}$ is $0.58(6) \mathrm{E}-27$, at $4 \mathrm{GeV}$ is $1.36(9) \mathrm{E}-27$ and at $8 \mathrm{GeV}$ is 2.92(14)E-27. These numbers is the fission reaction rates $R(n, f)$ for ${ }^{232} \mathrm{Th}$.

On the experimental results values of ratio $(\mathrm{n}, \gamma) /(\mathrm{n}, \mathrm{f})$ for different energies of deuterons: $2 \mathrm{GeV}-16.4(24)$, at $4 \mathrm{GeV}-13.0(20)$, at $8 \mathrm{GeV}-14.8(22)$. Calculated values (Cal.2.) for these ratios $(\mathrm{n}, \gamma) /(\mathrm{n}, \mathrm{f})$ are at $2 \mathrm{GeV}-12.3$, at $4 \mathrm{GeV}-12.2$ and at $8 \mathrm{GeV}-11.2$. 
Table.2. Values of the reaction rates ${ }^{232} \mathrm{Th}$ with secondary neutrons for product nuclei at energies of deuterons $2,4,8 \mathrm{GeV}$. (*) denotes mixing due to other nuclide.

\begin{tabular}{|c|c|c|c|c|c|c|c|}
\hline \multirow{2}{*}{$\begin{array}{c}\text { Isotope } \\
\text { Energy } \\
{[\mathrm{keV}]}\end{array}$} & \multirow{2}{*}{$\begin{array}{c}\mathrm{I}_{\mathrm{g}} \\
{[\%]}\end{array}$} & \multicolumn{2}{|c|}{$2 \mathrm{GeV}$} & \multicolumn{2}{|c|}{$4 \mathrm{GeV}$} & \multicolumn{2}{|c|}{$8 \mathrm{GeV}$} \\
\hline & & $\begin{array}{c}\mathbf{T}_{\mathbf{1 / 2} 2} \text { (Library) } \\
\mathrm{T}_{1 / 2} \text { (Exper.) }\end{array}$ & $\begin{array}{c}\langle\mathrm{R}> \\
\mathrm{R}\end{array}$ & $\begin{array}{c}\mathbf{T}_{\mathbf{1} / 2} \text { (Library) } \\
\mathrm{T}_{1 / 2} \text { (Exper.) }\end{array}$ & $\begin{array}{c}\langle\mathbf{R}> \\
\mathrm{R}\end{array}$ & $\begin{array}{c}\mathbf{T}_{\mathbf{1} / 2} \text { (Library) } \\
\mathrm{T}_{1 / 2} \text { (Exper.) }\end{array}$ & $\begin{array}{c}\langle\mathrm{R}> \\
\mathrm{R}\end{array}$ \\
\hline Ga-66 & & & & $9.49(7) \mathrm{h}$ & & $9.49(7) \mathrm{h}$ & \\
\hline 1039.231 & 37 & & & $12.6(20) \mathrm{h}$ & $2.68(62) E-29$ & $6(5) \mathrm{h}$ & $1.05(23) E-28$ \\
\hline Kr-85m & & $4.48(1) h$ & & $4.48(1) h$ & & & \\
\hline 151.159 & 75 & $7(3) d$ & $1.47(48)$ E-29 & & & & \\
\hline 304.870 & 14 & & & $3.2(11) \mathrm{h}$ & $5.52(85)$ E-29 & & \\
\hline Kr-87 & & $76.3(6) \mathrm{m}$ & & $76.3(6) \mathrm{m}$ & & $76.3(6) \mathrm{m}$ & \\
\hline 402.586 & 49.6 & $1.08(12) \mathrm{h}$ & 4.32(47)E-29 & $1.29(19) \mathrm{h}$ & $1.02(7) E-28$ & $1.7(7) \mathrm{h}$ & $2.64(69)$ E-28 \\
\hline Kr-88 & & $2.84(3) \mathrm{h}$ & $2.90(23) \mathrm{E}-29$ & $2.84(3) \mathrm{h}$ & 8.61(94)E-29 & $2.84(3) h$ & \\
\hline 196.301 & 26 & $2.3(4) \mathrm{h}$ & $2.85(25) \mathrm{E}-29$ & & & & \\
\hline 1529.770 & 10.9 & & & $3.9(1) \mathrm{h}$ & $1.08(11) \mathrm{E}-28$ & $12(5) \mathrm{h}$ & 3.2(11)E-28 \\
\hline 2195.842 & 13.2 & & $3.36(71) \mathrm{E}-29$ & & $7.4(10) \mathrm{E}-29$ & & \\
\hline 2392.110 & 34.6 & $2.5(22) \mathrm{h}$ & $3.22(95) \mathrm{E}-29$ & $2.11(22) \mathrm{h}$ & $8.17(93)$ E-29 & & \\
\hline Y-88 & & & & $106.65(4) d$ & & $106.65(4) d$ & \\
\hline 898.042 & 93.7 & & & & & & \\
\hline 1836.063 & 99.2 & & & & $2.99(36) \mathrm{E}-28$ & & 1.11(18)E-27 \\
\hline Y-91mD & & 49.71(4) $\mathrm{m}$ & & 49.71(4) $\mathrm{m}$ & & 49.71(4) $\mathrm{m}$ & \\
\hline 555.570 & 95 & $10.3(12) \mathrm{h}$ & $2.78(68)$ E-29 & & 1.61(9)E-28 & & $1.74(27) E-28$ \\
\hline Sr-91 & & $9.63(5) h$ & $4.00(90) E-29$ & $9.63(5) h$ & $1.01(3)$ E-28 & $9.63(5) h$ & $1.89(29) \mathrm{E}-28$ \\
\hline 652.900 & 8 & & & $14(10) \mathrm{h}$ & $1.15(11) \mathrm{E}-28$ & & \\
\hline 749.800 & 23.6 & $10.8(10) \mathrm{h}$ & $4.85(25) \mathrm{E}-29$ & $9.1(7) \mathrm{h}$ & $1.01(4) \mathrm{E}-28$ & $1.15(25) \mathrm{d}$ & $2.09(31) \mathrm{E}-28$ \\
\hline 1024.300 & 33 & $8.8(15) \mathrm{h}$ & $2.82(29) \mathrm{E}-29$ & $9.9(4)$ h 7 & $1.01(3) \mathrm{E}-28$ & $9.1(16) \mathrm{h}$ & $1.83(26) \mathrm{E}-28$ \\
\hline Sr-92 & & 2.71(1) h & & 2.71(1) h & & 2.71(1) h & \\
\hline 1383.930 & 90 & $2.62(10) \mathrm{h}$ & 3.81(16)E-29 & $2.68(11) \mathrm{h}$ & 8.81(32)E-29 & $2.74(27) \mathrm{h}$ & 1.82(13)E-28 \\
\hline Y-92 & & & & $3.54(1) \mathrm{h}$ & $1.45(13) E-28$ & 3.54(1) h & 4.7(10)E-28 \\
\hline 934.460 & 13.9 & & & & $1.63(36) \mathrm{E}-28$ & & $4.4(14) \mathrm{E}-28$ \\
\hline 1405.280 & 4.8 & & & $5.1(12) \mathrm{h}$ & $1.42(14) \mathrm{E}-28$ & & $4.9(16) \mathrm{E}-28$ \\
\hline
\end{tabular}




\begin{tabular}{|c|c|c|c|c|c|c|c|}
\hline Nb-92m & & & & $10.15(2) d$ & & $10.15(2) d$ & \\
\hline 934.460 & 99 & & & & 2.43(19)E-28 & & $3.16(69) \mathrm{E}-28$ \\
\hline Y-93 & & $10.18(8) h$ & & & & & \\
\hline 266.900 & 7.3 & $9(3) \mathrm{h}$ & $4.28(56) \mathrm{E}-29$ & & & & \\
\hline Zr-97 & & $16.91(5) \mathrm{h}$ & & $16.91(5) \mathrm{h}$ & & $16.91(5) \mathrm{h}$ & \\
\hline 743.360 & 93 & $14.7(8) \mathrm{h}$ & $2.88(14) E-29$ & $15.7(6) \mathrm{h}$ & $6.76(18) \mathrm{E}-29$ & $17.2(24) \mathrm{h}$ & $1.26(9) \mathrm{E}-28$ \\
\hline Nb-97 & & & & 72.1(7) $\mathrm{m}$ & & & \\
\hline 658.080 & 98 & & & & 6.89(46)E-29 & & \\
\hline Ru-105 & & 4.44(2) $h$ & & $4.44(2) h$ & $2.66(28) \mathrm{E}-29$ & 4.44(2) h & \\
\hline 469.370 & 17.5 & & & & $2.03(78) \mathrm{E}-29$ & & \\
\hline 724.210 & 47 & $9(6) \mathrm{h}$ & 1.15(11)E-29 & $5.7(26) \mathrm{h}$ & $2.74(30) \mathrm{E}-29$ & & 8.9(22)E-29 \\
\hline Cd-115 & & & & $53.46(1) \mathrm{h}$ & & $53.46(1) h$ & \\
\hline 336.240 & 45.9 & & & & & & \\
\hline 527.900 & 27.5 & & & $1.7(5) \mathrm{d}$ & $3.88(62) E-29$ & & $1.98(65) \mathrm{E}-28$ \\
\hline In-115m & & 4.87(1) $h$ & & & & & \\
\hline 336.240 & 45.8 & $4.4(20) \mathrm{h}$ & 8.4(15)E-30 & & & & \\
\hline In-117D & & 43.2(3) $\mathrm{m}$ & $1.90(34) E-29$ & 43.2(3) $\mathrm{m}$ & & 43.2(3) $\mathrm{m}$ & \\
\hline 158.562 & 87 & & $1.69(37) \mathrm{E}-29$ & & & & \\
\hline 553.000 & 100 & & $2.12(32) \mathrm{E}-29$ & & 7.26(95)E-29 & $26.5(1) \mathrm{m}$ & 1.04(27)E-28 \\
\hline Sb-126 & & & & $12.46(3) \mathrm{d}$ & 4.81(51)E-29 & $12.46(3) \mathrm{d}$ & $3.74(52) \mathrm{E}-28$ \\
\hline 414.810 & 83.3 & & & & & & \\
\hline 666.331 & 100 & & & & $4.0(11) \mathrm{E}-29$ & & $4.38(77) \mathrm{E}-28$ \\
\hline 695.030 & 100 & & & & $5.06(58) \mathrm{E}-29$ & & $3.03(88) \mathrm{E}-28$ \\
\hline Sn-127 & & & & & & 91.1(5) m & \\
\hline 1114.300 & 39 & & & & & $2.08(1) \mathrm{h}$ & $6.9(20) E-29$ \\
\hline $\mathrm{Sb}-128$ & & 9.01(3) h & & 9.01(3) h & & 9.01(3) h & $7.1(16) \mathrm{E}-29$ \\
\hline 314.120 & 61 & & & & & & $4.6(23) \mathrm{E}-29$ \\
\hline 526.570 & 45 & & & $4.5(22) \mathrm{h}$ & $2.76(21) E-29$ & & $7.9(22) \mathrm{E}-29$ \\
\hline 743.220 & 100 & $14.7(8) \mathrm{h}$ & $2.08(10)$ E-29 & & $5.56(59) \mathrm{E}-29 *$ & & $8.0(14) \mathrm{E}-29$ \\
\hline Sb-129 & & & & $4.40(1) h$ & & & \\
\hline 812.800 & 43 & & & $6(4) \mathrm{h}$ & 7.9(16)E-30 & & \\
\hline Te-132 & & & & $3.20(1) \mathrm{d}$ & & $3.20(1) \mathrm{d}$ & \\
\hline
\end{tabular}




\begin{tabular}{|c|c|c|c|c|c|c|c|}
\hline 228.160 & 88 & & & $15(10) \mathrm{d}$ & 4.64(94)E-29 & & $1.46(38) E-28$ \\
\hline I-132 & & & & 2.29(1) h & $1.49(65) E-29$ & & \\
\hline 667.718 & 99 & & & & $1.35(77) \mathrm{E}-29$ & & \\
\hline 954.550 & 17.6 & & & & $1.81(70) \mathrm{E}-29$ & & \\
\hline I-133 & & $20.8(1) h$ & & $20.8(1) h$ & & $20.8(1) h$ & \\
\hline 529.872 & 87 & $1.06(15) \mathrm{d}$ & 2.02(14)E-29 & $21.2(2) \mathrm{h}$ & $6.49(20) \mathrm{E}-29$ & $1.24(15) \mathrm{d}$ & 1.27(9)E-28 \\
\hline I-134 & & $52.5(2) \mathrm{m}$ & & $52.5(2) \mathrm{m}$ & $1.69(11) \mathrm{E}-28$ & $52.5(2) \mathrm{m}$ & $2.62(27) \mathrm{E}-28$ \\
\hline 847.025 & 95.4 & $1.15(7) \mathrm{h}$ & $5.75(76)$ E-29 & $1.06(2) \mathrm{h}$ & $1.53(17) \mathrm{E}-28$ & $1.33(19) \mathrm{h}$ & $2.51(57) \mathrm{E}-28$ \\
\hline 884.090 & 64.9 & & & $1.11(7) \mathrm{h}$ & $1.59(18) \mathrm{E}-28$ & & $2.57(32) \mathrm{E}-28$ \\
\hline 1072.550 & 14.9 & & & $1.12(2) \mathrm{h}$ & 1.94(21)E-28 & & $3.4(13) \mathrm{E}-28$ \\
\hline 1136.160 & 9.1 & & & & $1.98(33) \mathrm{E}-28$ & & \\
\hline I-135 & & $6.57(2) \mathrm{h}$ & 3.09(14)E-29 & 6.57(2) h & 7.04(26)E-29 & 6.57(2) h & $1.72(28) E-28$ \\
\hline 1131.511 & 22.7 & $6.8(8) \mathrm{h}$ & $3.11(21) \mathrm{E}-29$ & $5.7(6) \mathrm{h}$ & $6.99(52) \mathrm{E}-29$ & $12.9(1) \mathrm{h}$ & $1.77(26) \mathrm{E}-28$ \\
\hline 1260.409 & 28.9 & $7(5) \mathrm{h}$ & $3.08(18) \mathrm{E}-29$ & $6.7(4) \mathrm{h}$ & $6.89(28) \mathrm{E}-29$ & $7(2) \mathrm{h}$ & $1.67(73) \mathrm{E}-28$ \\
\hline 1791.196 & 7.8 & & & $8.1(16) \mathrm{h}$ & $7.29(70) \mathrm{E}-29$ & & \\
\hline Xe-135 & & $9.14(2) h$ & & 9.14(2) h & & $9.14(2) \mathrm{h}$ & \\
\hline 249.770 & 90 & $16.8(21) \mathrm{h}$ & $2.85(55) E-29$ & $16.8(19) \mathrm{h}$ & $7.8(12) E-29$ & $20(3) \mathrm{h}$ & $1.95(34) \mathrm{E}-28$ \\
\hline Cs-138 & & & & 33.4(2) $\mathrm{m}$ & & 33.4(2) $\mathrm{m}$ & \\
\hline 1435.795 & 76.3 & & & & 1.31(13)E-28 & & $2.03(43) E-28$ \\
\hline La-142 & & 91.1(5) m & & 91.1(5) m & 7.73(82)E-29 & 91.1(5) m & \\
\hline 641.285 & 47 & $1.86(19) \mathrm{h}$ & 3.91(31)E-29 & $1.9(5) \mathrm{h}$ & $8.4(13) \mathrm{E}-29$ & $2.08(1) \mathrm{h}$ & $1.66(24) \mathrm{E}-28$ \\
\hline 894.900 & 8.3 & & & $3.46(2) \mathrm{h}$ & $7.3(14) \mathrm{E}-29$ & & \\
\hline 2397.800 & 13.3 & & & & $7.3(15) \mathrm{E}-29$ & & \\
\hline Ce-143 & & $1.38(2) \mathrm{d}$ & & $1.38(2) \mathrm{d}$ & & $1.38(2) \mathrm{d}$ & \\
\hline 293.266 & 42.8 & $1.68(3) \mathrm{d}$ & 3.32(22)E-29 & $1.50(18) \mathrm{d}$ & $7.80(35) \mathrm{E}-29$ & $3.1(7) \mathrm{d}$ & 2.51(35)E-28 \\
\hline At-208 & & & & $1.63(3) \mathrm{h}$ & & $1.63(3) h$ & \\
\hline 177.595 & 48.6 & & & & & & 6.1(23)E-28 \\
\hline 845.044 & 19.7 & & & & $3.83(64) E-29$ & & \\
\hline Ac-224 & & & & $2.78(17) h$ & & $2.78(17) h$ & \\
\hline 131.613 & 26.9 & & & & & & $1.13(51) \mathrm{E}-26$ \\
\hline 215.983 & 52.3 & & & & $2.84(80)$ E-29 & & \\
\hline Pa-233 & & 26.97(1)d & & 26.97(1) d & & 26.97(1) d & \\
\hline
\end{tabular}




\section{RESULTS OF ${ }^{129} \mathbf{I}$}

During irradiation the samples ${ }^{129} \mathrm{I}$ (coated aluminum weighing $17.6 \mathrm{~g}$ ) and ${ }^{127} \mathrm{I}$ (in a shell made of plexiglass weighing $2.53 \mathrm{~g}$ ) were installed on the side of the section №3 uranium assembly Fig.2. In samples ${ }^{129} \mathrm{I}$ impurity present stable isotope ${ }^{127}$ I. To correct account the contribution of ${ }^{127} \mathrm{I}$, samples ${ }^{129} \mathrm{I}$ were irradiated simultaneously with the samples, containing only isotope ${ }^{127} \mathrm{I}$.

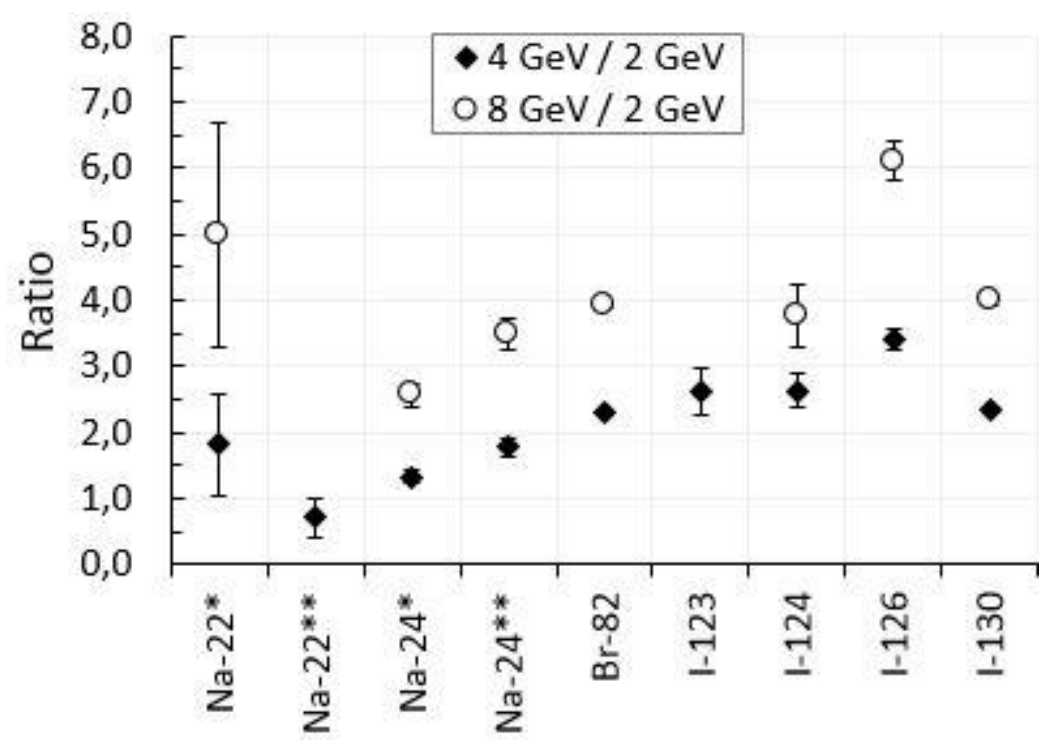

Fig.6. Experimental values of the ratio of reaction rate $\mathrm{R}(4 \mathrm{GeV}) / \mathrm{R}(2 \mathrm{GeV})$ and $\mathrm{R}(8 \mathrm{GeV}) / \mathrm{R}(2$ $\mathrm{GeV}$ ) for ${ }^{23} \mathrm{Na}+{ }^{27} \mathrm{Al}+{ }^{129} \mathrm{I}$ with secondary neutrons for product nuclei at energies of deuterons 2 , 4, $8 \mathrm{GeV} . \mathrm{Na}-22 *$ - product ${ }^{23} \mathrm{Na}(\mathrm{n}, 2 \mathrm{n})^{22} \mathrm{Na}$. Na-22** - product ${ }^{27} \mathrm{Al}(\mathrm{n}, \alpha 2 \mathrm{n})^{22} \mathrm{Na}$. Na-24* product ${ }^{23} \mathrm{Na}(\mathrm{n}, \gamma){ }^{24} \mathrm{Na}$. $\mathrm{Na}-24 * *$ - product ${ }^{27} \mathrm{Al}(\mathrm{n}, \alpha)^{24} \mathrm{Na}$. Values of reaction rates for ${ }^{82} \mathrm{Br}$ obtained from calculation Calc. 2 due to lack of weight values ${ }^{81} \mathrm{Br}$.

${ }^{22} \mathrm{Na}$ produced simultaneously in two reactions ${ }^{27} \mathrm{Al}(\mathrm{n}, \alpha 2 \mathrm{n}){ }^{22} \mathrm{Na}\left(\mathrm{E}_{\mathrm{thr}}=23.35 \mathrm{MэB}\right)$ and ${ }^{23} \mathrm{Na}(\mathrm{n}, 2 \mathrm{n}){ }^{22} \mathrm{Na} \quad\left(\mathrm{E}_{\mathrm{thr}}=12.96 \mathrm{MэB}\right) .{ }^{24} \mathrm{Na}$ is generated from ${ }^{27} \mathrm{Al}(\mathrm{n}, \alpha){ }^{24} \mathrm{Na}$ $\left(\mathrm{E}_{\text {thr }}=3.25 \mathrm{MэB}\right)$ and ${ }^{23} \mathrm{Na}(\mathrm{n}, \gamma)^{24} \mathrm{Na}$ reactions. Share ${ }^{24} \mathrm{Na}$ generated from the $(\mathrm{n}, \gamma)$ reaction at deuteron energies $2 \mathrm{GeV}-2.1 \%, 4 \mathrm{GeV}-0.9 \%$ and $8 \mathrm{GeV}-0.6 \%$.

${ }^{22} \mathrm{Na}$ and ${ }^{24} \mathrm{Na}$ produced mainly from ${ }^{27} \mathrm{Al}$ due to large mass of ${ }^{27} \mathrm{Al}$. Contribution ${ }^{24} \mathrm{Na}$ produced from ${ }^{23} \mathrm{Na}$ calculated using the values of reaction rates for ${ }^{24} \mathrm{Na}$ in the sample ${ }^{127}$ I.

We assume that in the composition of samples ${ }^{129} \mathrm{I}$, present admixture of ${ }^{81} \mathrm{Br}$ and ${ }^{82} \mathrm{Br}$ is a product of ${ }^{81} \mathrm{Br}(\mathrm{n}, \gamma){ }^{82} \mathrm{Br}$ reaction. The admixture of ${ }^{81} \mathrm{Br}$ may be, by our estimates (Calc.2), in ${ }^{129} \mathrm{I}$ no more than $1.5(5) \% .{ }^{82} \mathrm{Br}$ in the samples ${ }^{127} \mathrm{I}$ was not observed. ${ }^{123} \mathrm{I},{ }^{124} \mathrm{I}$, and ${ }^{126} \mathrm{I}$ are products of $(\mathrm{n}, 7 \mathrm{n}),(\mathrm{n}, 6 \mathrm{n})$ and $(\mathrm{n}, 4 \mathrm{n})$ reactions. ${ }^{130} \mathrm{I}$ is product $(n, \gamma)$ reaction. 


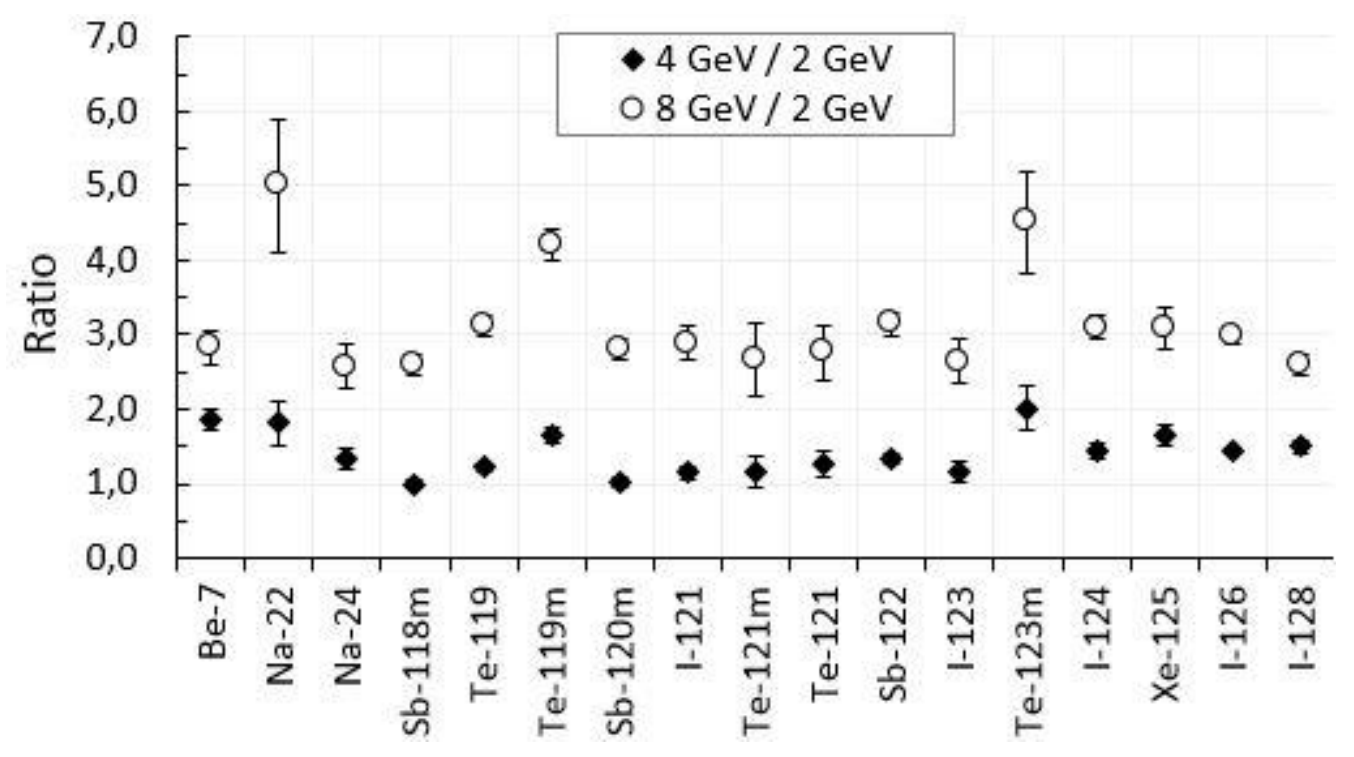

Fig.7. Experimental values of the ratio of reaction rate $\mathrm{R}(4 \mathrm{GeV}) / \mathrm{R}(2 \mathrm{GeV})$ and $\mathrm{R}(8 \mathrm{GeV}) / \mathrm{R}(2$ $\mathrm{GeV}$ ) for ${ }^{23} \mathrm{Na}+{ }^{127} \mathrm{I}$ with secondary neutrons for product nuclei at energies of deuterons $2,4,8$ $\mathrm{GeV}$.

${ }^{7} \mathrm{Be}$ is produced in the shell of the sample (plexiglass) from ${ }^{12} \mathrm{C}$ and ${ }^{16} \mathrm{O} \cdot{ }^{22} \mathrm{Na}$ and ${ }^{24} \mathrm{Na}$ produced from ${ }^{23} \mathrm{Na}$ in the reactions $(\mathrm{n}, 2 \mathrm{n})$ and $(\mathrm{n}, \gamma) \cdot{ }^{118 \mathrm{~m}} \mathrm{Sb},{ }^{120 \mathrm{~m}} \mathrm{Sb}$ and ${ }^{122} \mathrm{Sb}$ are products of $(n, \alpha 6 n)\left(E_{t h r}=44.11 \mathrm{MeV}\right),(n, \alpha 4 n)\left(E_{t h r}=27.42 \mathrm{MeV}\right)$ and $(n, \alpha 2 n)$ $\left(\mathrm{E}_{\mathrm{thr}}=11.23 \mathrm{MeV}\right)$ reactions. Radionuclides ${ }^{119} \mathrm{Te},{ }^{121} \mathrm{Te}$ and ${ }^{123 \mathrm{~m}} \mathrm{Te}$ are products of $(\mathrm{n}, \mathrm{t} 6 \mathrm{n})\left(\mathrm{E}_{\mathrm{thr}}=57.56 \mathrm{MeV}\right),(\mathrm{n}, \mathrm{t} 4 \mathrm{n})\left(\mathrm{E}_{\mathrm{thr}}=39.92 \mathrm{MeV}\right)$ and $(\mathrm{n}, \mathrm{t} 2 \mathrm{n})\left(\mathrm{E}_{\mathrm{thr}}=23.01 \mathrm{MeV}\right)$. ${ }^{120} \mathrm{I},{ }^{121} \mathrm{I},{ }^{123} \mathrm{I},{ }^{124} \mathrm{I}$, and ${ }^{126} \mathrm{I}$ are products of $(\mathrm{n}, 8 \mathrm{n})\left(\mathrm{E}_{\mathrm{thr}}=62.18 \mathrm{MeV}\right),(\mathrm{n}, 7 \mathrm{n})\left(\mathrm{E}_{\mathrm{thr}}=51.53\right.$ $\mathrm{MeV}),(\mathrm{n}, 5 \mathrm{n})\left(\mathrm{E}_{\mathrm{thr}}=33.59 \mathrm{MeV}\right),(\mathrm{n}, 4 \mathrm{n})\left(\mathrm{E}_{\mathrm{thr}}=26.01 \mathrm{MeV}\right)$ and $(\mathrm{n}, 2 \mathrm{n})\left(\mathrm{E}_{\mathrm{thr}}=44.11\right.$ $\mathrm{MeV})$ reactions. ${ }^{128} \mathrm{I}$ is product $(\mathrm{n}, \gamma)$ reaction. Table 2 summarizes the results of comparing the experimental and calculated data (Calc.1 and Calc.2) on ${ }^{127} \mathrm{I}$ and ${ }^{129} \mathrm{I}$.

Table.2. Comparison of results for ${ }^{127} \mathrm{I}$ and ${ }^{129} \mathrm{I}$ with calculations.

\begin{tabular}{|c|c|c|c|c|c|c|}
\hline \multirow{2}{*}{$\begin{array}{c}\text { Nuclear } \\
\text { reactions } \\
\text { on }{ }^{127} \mathbf{I} \\
\text { samples }\end{array}$} & \multicolumn{2}{|c|}{$2 \mathrm{GeV}$} & \multicolumn{2}{|c|}{$4 \mathrm{GeV}$} & \multicolumn{2}{|c|}{$8 \mathrm{GeV}$} \\
\hline & $\begin{array}{c}\text { Exp/Calc. } \\
1\end{array}$ & $\begin{array}{c}\text { Exp/Calc. } \\
2\end{array}$ & $\begin{array}{c}\text { Exp/Calc. } \\
1\end{array}$ & $\begin{array}{c}\text { Exp/Calc. } \\
2\end{array}$ & $\begin{array}{c}\text { Exp/Calc. } \\
1\end{array}$ & $\begin{array}{c}\text { Exp/Calc. } \\
2\end{array}$ \\
\hline${ }^{127} \mathbf{I}(\mathbf{n}, \gamma)^{128} \mathbf{I}$ & & $0.64(4)$ & & $0.52(3)$ & & $0.53(3)$ \\
\hline $\begin{array}{c}{ }^{127} I(n, 2 n)^{126} \\
I\end{array}$ & $1.10(4)$ & $0.66(2)$ & $0.85(3)$ & $0.57(2)$ & $0.69(3)$ & $0.53(1)$ \\
\hline $\begin{array}{c}{ }^{127} I(n, 4 n)^{124} \\
I\end{array}$ & $1.18(9)$ & $0.68(2)$ & $0.89(8)$ & $0.64(7)$ & $0.79(7)$ & $0.62(4)$ \\
\hline $\begin{array}{c}\text { Nuclear } \\
\text { reactions } \\
\text { on }{ }^{129} \text { I } \\
\text { samples }\end{array}$ & & & & & & \\
\hline${ }^{129} \mathrm{I}(\mathrm{n}, \gamma)^{130} \mathrm{I}$ & & $0.61(1)$ & & $0.71(1)$ & & $0.71(1)$ \\
\hline $\begin{array}{c}{ }^{129} I(n, 4 n)^{126} \\
I\end{array}$ & $1.45(12)$ & $0.72(3)$ & $2.46(17)$ & $1.56(6)$ & $1.74(27)$ & $1.27(6)$ \\
\hline
\end{tabular}




\begin{tabular}{|c|c|c|c|c|c|c|}
\hline $\begin{array}{c}\mathbf{1 2 9}^{\mathbf{I}}(\mathbf{n , 6 n})^{\mathbf{1 2 4}} \\
\mathbf{I}\end{array}$ & $1.95(26)$ & $0.88(5)$ & $2.49(32)$ & $1.49(19)$ & $1.43(23)$ & $0.94(18)$ \\
\hline
\end{tabular}

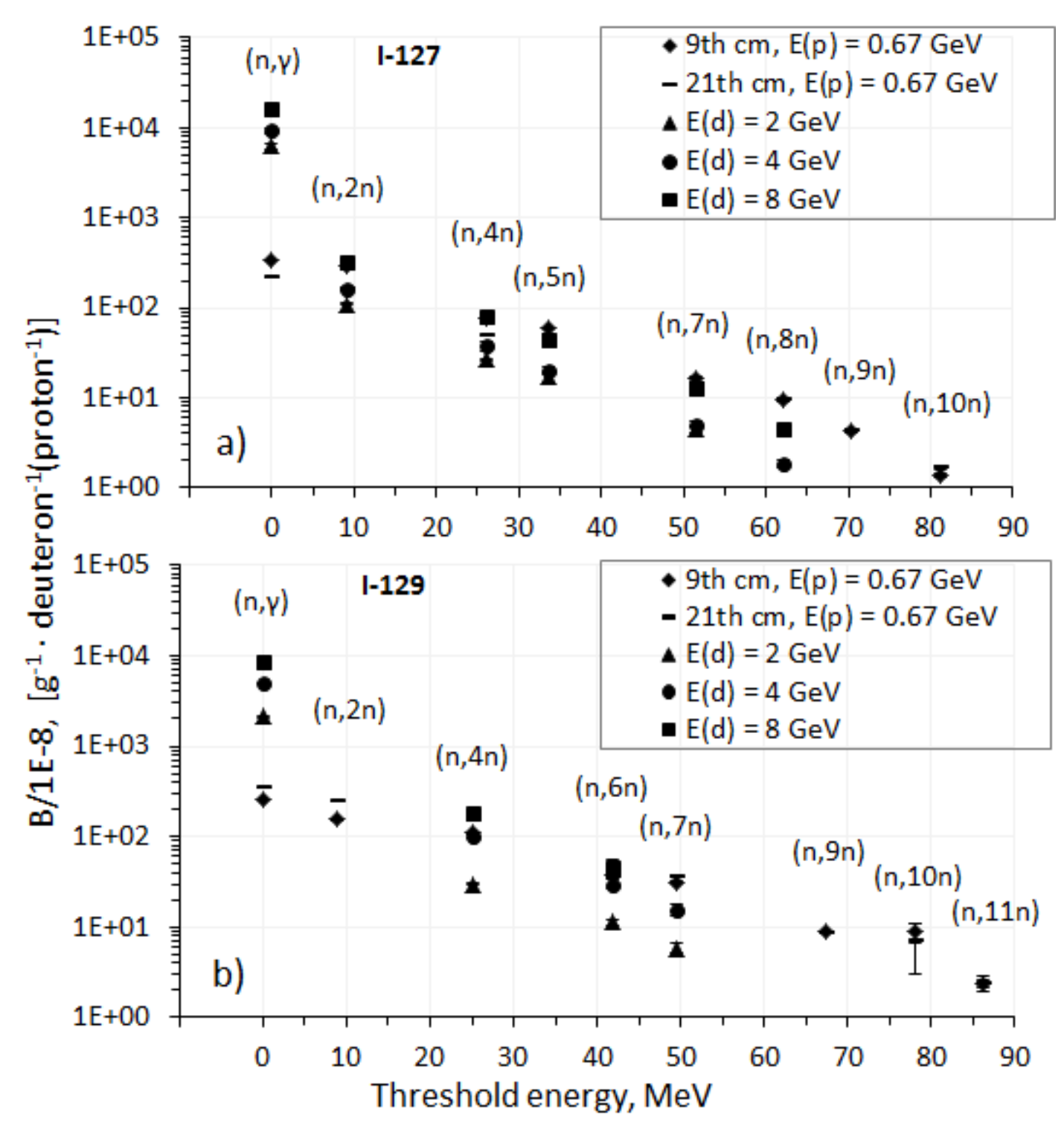

Fig.8. Comparison of the experimental results on ${ }^{127} \mathrm{I}-\mathrm{a}$ ) and ${ }^{129} \mathrm{I}-\mathrm{b}$ ) with [18].

Previously, we performed an experiment [18], in which for production of secondary neutrons used massive lead assembly installed in the proton beam (660 $\mathrm{MeV}$ ) in Phasotron DLNP. Data for ${ }^{127} \mathrm{I}$ and ${ }^{129} \mathrm{I}$, obtained in this experiment, we compare with data from experiments at the Nuclotron VBLHEP (see Fig.8). It can be seen that the results for ${ }^{127} \mathrm{I}$ and ${ }^{129} \mathrm{I}$ in good agreement for almost all the observed $(n, x n)$ reactions (in this work have not been identified $(n, 9 n),(n, 10 n)$ reactions in 
127I, (n, 9n), (n, 10n) and $(n, 11 n)$ reactions in 129I, as the time from end of irradiation up to start of measurement $80-160 \mathrm{~min}$.), except the (n, $\gamma$ ) reaction, which is caused by the presence on setup "QUINTA" lead shield that reflects neutrons. 
Table.3. Values of the reaction rates ${ }^{127} \mathrm{I}$ with secondary neutrons for product nuclei at energies of deuterons $2,4,8 \mathrm{GeV}$. (*) denotes mixing due to other nuclide.

\begin{tabular}{|c|c|c|c|c|c|c|c|}
\hline \multirow{2}{*}{$\begin{array}{c}\text { Isotope } \\
\text { Energy } \\
{[\mathrm{keV}]}\end{array}$} & \multirow[b]{2}{*}{$\begin{array}{c}\mathrm{Ig}_{\mathrm{g}} \\
{[\%]}\end{array}$} & \multicolumn{2}{|c|}{$2 \mathrm{GeV}$} & \multicolumn{2}{|c|}{$4 \mathrm{GeV}$} & \multicolumn{2}{|c|}{$8 \mathrm{GeV}$} \\
\hline & & $\begin{array}{c}\mathbf{T}_{\mathbf{1} / 2} \text { (Library) } \\
\mathrm{T}_{1 / 2} \text { (Exper.) }\end{array}$ & $\begin{array}{c}<\mathrm{R}> \\
\mathrm{R}\end{array}$ & $\begin{array}{c}\mathbf{T}_{\mathbf{1} / 2} \text { (Library) } \\
\mathrm{T}_{1 / 2} \text { (Exper.) }\end{array}$ & $\begin{array}{c}<\mathrm{R}> \\
\mathrm{R}\end{array}$ & $\begin{array}{c}\mathbf{T}_{\mathbf{1} / 2} \text { (Library) } \\
\mathrm{T}_{1 / 2} \text { (Exper.) }\end{array}$ & $\begin{array}{c}\langle\mathbf{R}> \\
\mathrm{R}\end{array}$ \\
\hline Be-7 & & $53.12(7) \mathrm{d}$ & & $53.12(7) d$ & & $53.12(7) d$ & \\
\hline 477.595 & 10.5 & $38(15) \mathrm{d}$ & 3.68(30)E-29 & $25(12) \mathrm{d}$ & $6.81(47) \mathrm{E}-29$ & & 1.04(9)E-28 \\
\hline Na-22 & & $2.60(1) y$ & & $2.60(1) y$ & & $2.60(1) y$ & \\
\hline 1274.530 & 99.94 & $100(110) \mathrm{d}$ & $5.58(51)$ E-29 & & 1.01(24)E-28 & $7.0(15) \mathrm{d}$ & 2.79(75)E-28 \\
\hline $\mathrm{Na}-24$ & & $14.96(1) h$ & $7.22(66)$ E-29 & $14.96(1) h$ & 9.6(11)E-29 & $14.96(1) h$ & $1.85(26) E-28$ \\
\hline 1368.633 & 100 & $15.18(18) \mathrm{h}$ & $7.04(15) \mathrm{E}-29$ & $15.21(10) \mathrm{h}$ & $9.40(24) \mathrm{E}-29$ & $15.17(14) \mathrm{h}$ & $1.79(5) \mathrm{E}-28$ \\
\hline 2754.028 & 99.94 & $15.31(21) \mathrm{h}$ & $9.65(58) \mathrm{E}-29$ & $14.86(10) \mathrm{h}$ & $1.39(9) \mathrm{E}-28$ & $14.76(19) \mathrm{h}$ & 2.93(19)E-28 \\
\hline Ag-108m & & & & & & $418(21) y$ & $2.70(63) E-30$ \\
\hline 433.937 & 90 & & & & & & $2.57(73) \mathrm{E}-30$ \\
\hline 614.276 & 89.8 & & & & & & $2.85(76) \mathrm{E}-30$ \\
\hline 722.907 & 90.8 & & & & & & $6.9(13) \mathrm{E}-30 *$ \\
\hline In-111 & & $2.80(1) \mathrm{d}$ & 5.87(72)E-31 & & & & \\
\hline 171.280 & 90 & $2.8(4) \mathrm{d}$ & $5.26(40) \mathrm{E}-31$ & & & & \\
\hline 245.395 & 94 & $2.5(4) d$ & $6.73(47) \mathrm{E}-31$ & & & & \\
\hline Sb-118m & & $5.00(2) h$ & $1.55(6) \mathrm{E}-30$ & $5.00(2) h$ & 1.54(11)E-30 & $5.00(2) h$ & 4.01(30)E-30 \\
\hline 253.678 & 99 & $5.09(25) \mathrm{h}$ & 1.47(7)E-30 & $5.1(4) \mathrm{h}$ & 1.44(9)E-30 & $5.0(4) \mathrm{h}$ & $3.44(21) \mathrm{E}-30$ \\
\hline 1050.650 & 97 & $5.8(7) \mathrm{h}$ & $1.64(11) \mathrm{E}-30$ & $4.6(7) \mathrm{h}$ & $1.76(16) \mathrm{E}-30$ & $4.7(4) \mathrm{h}$ & $4.51(35) \mathrm{E}-30$ \\
\hline 1229.680 & 100 & $5.1(4) \mathrm{h}$ & $1.62(10) \mathrm{E}-30$ & $8.3(14) \mathrm{h}$ & $1.73(23) \mathrm{E}-30$ & $6.2(8) \mathrm{h}$ & $4.26(41) \mathrm{E}-30$ \\
\hline Te-119 & & $16.03(5) h$ & & $16.03(5) h$ & & $16.03(5) h$ & \\
\hline 644.010 & 84 & $19.0(6) \mathrm{h}$ & 3.24(14)E-30 & $16.9(7) \mathrm{h}$ & 3.92(13)E-30 & $15.5(6) \mathrm{h}$ & 1.10(4)E-29 \\
\hline Te-119m & & $4.70(4) d$ & $2.49(16) \mathrm{E}-30$ & $4.70(4) d$ & $4.09(23) \mathrm{E}-30$ & $4.70(4) d$ & $1.05(4) E-29$ \\
\hline 153.590 & 66 & $4.9(4) d$ & $2.39(11) \mathrm{E}-30$ & $4.1(6) \mathrm{d}$ & $4.20(36) \mathrm{E}-30$ & $3.92(23) \mathrm{d}$ & $1.02(5) \mathrm{E}-29$ \\
\hline 1212.730 & 66 & $5.4(7) \mathrm{d}$ & $2.75(17) \mathrm{E}-30$ & $11.3(28) \mathrm{d}$ & $4.02(30) \mathrm{E}-30$ & $4.6(8) \mathrm{d}$ & $1.11(8) \mathrm{E}-29$ \\
\hline I-120 & & & & $81.0(6) \mathrm{m}$ & & $81.0(6) \mathrm{m}$ & \\
\hline 560.440 & 73 & & & $1.32(2) \mathrm{h}$ & 3.87(36)E-30 & $1.40(14) \mathrm{h}$ & 9.3(8)E-30 \\
\hline Sb-120m & & $5.76(2) d$ & $1.58(8) E-30$ & $5.76(2) d$ & 1.61(19)E-30 & $5.76(2) d$ & $4.43(24) \mathrm{E}-30$ \\
\hline
\end{tabular}




\begin{tabular}{|c|c|c|c|c|c|c|c|}
\hline 197.300 & 87 & $12.3(21) \mathrm{d}$ & $1.50(13) \mathrm{E}-30$ & $5.5(7) \mathrm{d}$ & $1.51(21) \mathrm{E}-30$ & $5.6(5) \mathrm{d}$ & $3.83(33) \mathrm{E}-30$ \\
\hline 1023.100 & 99.4 & $9(4) \mathrm{d}$ & $1.63(16) \mathrm{E}-30$ & $1.6(10) \mathrm{d}$ & $1.86(57) \mathrm{E}-30$ & $5.4(5) \mathrm{d}$ & 4.44(33)E-30 \\
\hline 1171.300 & 100 & $5.4(13) \mathrm{d}$ & $1.63(12) \mathrm{E}-30$ & & & $5.0(15) \mathrm{d}$ & $4.52(92) \mathrm{E}-30$ \\
\hline I-121 & & 2.12(1) h & & 2.12(1) h & & 2.12(1) h & \\
\hline 212.189 & 84 & $2.14(9) \mathrm{h}$ & 9.48(73)E-30 & $2.05(10) \mathrm{h}$ & 1.07(7)E-29 & $1.99(9) \mathrm{h}$ & $2.71(21) E-29$ \\
\hline Te-121m & & 154(7) d & & $154(7) d$ & & 154(7) d & \\
\hline 212.189 & 81 & & $8.7(16) \mathrm{E}-30$ & & $1.01(20) E-29$ & & $2.33(42) E-29$ \\
\hline Te-121 & & $16.78(35) d$ & $1.26(26)$ E-29 & $16.78(35) d$ & & $16.78(35) d$ & 3.47(20)E-29 \\
\hline 507.591 & 17.7 & $23(8) \mathrm{d}$ & $2.37(22) \mathrm{E}-29$ & & & $13(5) \mathrm{d}$ & $6.23(73) \mathrm{E}-29 *$ \\
\hline 573.139 & 80.3 & $22.7(10) \mathrm{d}$ & $1.20(5) \mathrm{E}-29$ & $19.3(13) \mathrm{d}$ & $1.60(11) E-29$ & $16.6(19) \mathrm{d}$ & $3.47(20) \mathrm{E}-29$ \\
\hline Sb-122 & & $2.72(1) \mathrm{d}$ & & $2.72(1) \mathrm{d}$ & & $2.72(1) \mathrm{d}$ & \\
\hline 564.119 & 71 & $2.64(23) \mathrm{d}$ & 2.29(11)E-30 & $2.4(3) \mathrm{d}$ & 3.08(16)E-30 & $2.89(22) \mathrm{d}$ & $7.19(36) \mathrm{E}-30$ \\
\hline I-123 & & 13.27(8) h & & 13.27(8) h & & 13.27(8) h & \\
\hline 158.970 & 83 & $13.0(6) \mathrm{h}$ & 3.59(41)E-29 & $12.3(6) \mathrm{h}$ & $4.18(45) E-29$ & $12.5(7) \mathrm{h}$ & $9.5(11) \mathrm{E}-29$ \\
\hline Te-123m & & 119.7(1)d & & 119.7(1) d & & $119.7(1) \mathrm{d}$ & \\
\hline 158.970 & 84 & & 7.7(13)E-30 & & $1.55(21) E-29$ & & 3.47(47)E-29 \\
\hline I-124 & & $4.18(2) d$ & $5.54(18)$ E-29 & $4.18(2) d$ & $7.93(85)$ E-29 & $4.18(2) d$ & 1.71(12)E-28 \\
\hline 602.729 & 63 & $4.18(15) \mathrm{d}$ & $5.56(15) \mathrm{E}-29$ & $4.13(5) \mathrm{d}$ & 7.29(30)E-29 & $3.96(9) \mathrm{d}$ & $1.56(5) \mathrm{E}-28$ \\
\hline 722.786 & 10.3 & $4.14(22) \mathrm{d}$ & $5.26(17) \mathrm{E}-29$ & $17(7) \mathrm{d}$ & $1.21(10) \mathrm{E}-28$ & $5.1(11) \mathrm{d}$ & $2.44(21) \mathrm{E}-28$ \\
\hline 1325.512 & 1.6 & $3.4(10) \mathrm{d}$ & $7.40(81)$ E-29 & $4.8(28) \mathrm{d}$ & $1.03(10) \mathrm{E}-28$ & $3.4(3) \mathrm{d}$ & $2.46(20) \mathrm{E}-28$ \\
\hline 1509.470 & 3.1 & & & $5.5(7) \mathrm{d}$ & $6.9(12) \mathrm{E}-29$ & $4.5(5) \mathrm{d}$ & $1.97(22) \mathrm{E}-28$ \\
\hline 1690.983 & 10.9 & $4.6(4) d$ & 5.84(23)E-29 & $4.9(3) \mathrm{d}$ & $7.02(39) \mathrm{E}-29$ & $4.07(17) \mathrm{d}$ & $1.63(6) \mathrm{E}-28$ \\
\hline Xe-125 & & $16.9(2) \mathrm{h}$ & $7.93(70) E-31$ & $16.9(2) \mathrm{h}$ & 1.31(10)E-30 & $16.9(2) \mathrm{h}$ & $2.45(22) \mathrm{E}-30$ \\
\hline 188.418 & 54 & $17.1(26) \mathrm{h}$ & 7.57(61)E-31 & $15.3(23) \mathrm{h}$ & $1.28(10) \mathrm{E}-30$ & $20(6) \mathrm{h}$ & $2.10(20) \mathrm{E}-30$ \\
\hline 243.378 & 30 & $1.8(5) \mathrm{d}$ & $9.3(12) \mathrm{E}-31$ & & 1.61(30)E-30 & & $2.89(63) \mathrm{E}-30$ \\
\hline I-126 & & 13.11(5) d & $2.32(7) E-28$ & $13.11(5) \mathrm{d}$ & 3.35(12)E-28 & 13.11(5) d & $6.90(18) \mathrm{E}-28$ \\
\hline 388.633 & 34.1 & $13.3(6) \mathrm{d}$ & $2.49(5) \mathrm{E}-28$ & $12.37(16) \mathrm{d}$ & $3.44(16) \mathrm{E}-28$ & $10.7(5) \mathrm{d}$ & $6.85(22) \mathrm{E}-28$ \\
\hline 491.243 & 2.8 & $12.8(6) \mathrm{d}$ & $2.33(8) \mathrm{E}-28$ & $11.8(6) \mathrm{d}$ & $3.27(18) \mathrm{E}-28$ & $11.1(11) \mathrm{d}$ & $6.44(28) \mathrm{E}-28$ \\
\hline 666.331 & 33.1 & $13.8(7) \mathrm{d}$ & $2.27(5) \mathrm{E}-28$ & $11.98(29) \mathrm{d}$ & $3.36(11) \mathrm{E}-28$ & $10.8(6) \mathrm{d}$ & $6.73(16) \mathrm{E}-28$ \\
\hline 753.819 & 4.2 & $14.0(7) \mathrm{d}$ & $2.16(6) \mathrm{E}-28$ & $11.5(6) \mathrm{d}$ & $3.14(17) \mathrm{E}-28$ & $11.0(11) \mathrm{d}$ & $6.53(25) \mathrm{E}-28$ \\
\hline 879.876 & 0.7 & $15(4) \mathrm{d}$ & $2.57(29) \mathrm{E}-28$ & $12.8(18) \mathrm{d}$ & $4.74(48) \mathrm{E}-28$ & $9.1(12) \mathrm{d}$ & $9.68(97) \mathrm{E}-28^{*}$ \\
\hline I-128 & & $24.99(2) \mathrm{m}$ & & 24.99(2) $\mathrm{m}$ & 1.95(12)E-26 & 24.99(2) $\mathrm{m}$ & 3.38(17)E-26 \\
\hline
\end{tabular}




\begin{tabular}{|l|l|l|l|l|l|l|l|}
\hline 442.901 & 17 & $25.2(1) \mathrm{m}$ & $\mathbf{1 . 3 0}(\mathbf{8}) \mathrm{E}-26$ & $25.06(2) \mathrm{m}$ & $1.97(12) \mathrm{E}-26$ & $24.90(2) \mathrm{m}$ & $3.45(6) \mathrm{E}-26$ \\
\hline 526.557 & 1.6 & & & $31.1(1) \mathrm{m}$ & $1.87(26) \mathrm{E}-26$ & $24.68(2) \mathrm{m}$ & $3.29(8) \mathrm{E}-26$ \\
\hline
\end{tabular}

Table.4. Values of the reaction rates ${ }^{129} \mathrm{I}$ with secondary neutrons for product nuclei at energies of deuterons $2,4,8 \mathrm{GeV}$.

(*) denotes mixing due to other nuclide.

\begin{tabular}{|c|c|c|c|c|c|c|c|}
\hline \multirow{3}{*}{$\begin{array}{c}\text { Isotope } \\
\text { Energy } \\
{[\mathrm{keV}]}\end{array}$} & \multirow{3}{*}{$\begin{array}{c}\mathrm{I}_{\mathrm{g}} \\
{[\%]}\end{array}$} & \multicolumn{2}{|c|}{$2 \mathrm{GeV}$} & \multicolumn{2}{|c|}{$4 \mathrm{GeV}$} & \multicolumn{2}{|c|}{$8 \mathrm{GeV}$} \\
\hline & & $T_{1 / 2}($ Library) & $\langle\mathbf{R}\rangle$ & $\mathbf{T}_{1 / 2}($ Library $)$ & $\langle\mathbf{R}\rangle$ & $\mathbf{T}_{1 / 2}($ Library $)$ & $\langle\mathbf{R}\rangle$ \\
\hline & & $\mathrm{T}_{1 / 2}$ (Exper.) & $\mathrm{R}$ & $\mathrm{T}_{1 / 2}($ Exper.) & $\mathrm{R}$ & $\mathrm{T}_{1 / 2}$ (Exper.) & $\mathrm{R}$ \\
\hline Na-22* & & $2.60(1) y$ & & $2.60(1) y$ & & $2.60(1) y$ & \\
\hline 1274.530 & 99.94 & $40(24) d$ & $5.6(34) \mathrm{E}-29$ & $40(24) d$ & 1.01(23)E-28 & & $2.79(20) E-28$ \\
\hline $\mathrm{Na}-22 * *$ & & $2.60(1) y$ & & $2.60(1) y$ & & $2.60(1) y$ & \\
\hline 1274.530 & 99.94 & $40(24) d$ & 8.1(49)E-30 & $40(24) d$ & $5.7(13) \mathrm{E}-30$ & & $6.42(46) \mathrm{E}-28$ \\
\hline Na-24* & & 14.96(1) h & $7.22(43)$ E-29 & $14.96(1) h$ & $9.60(79) \mathrm{E}-29$ & $14.96(1) \mathrm{h}$ & $1.85(14) E-28$ \\
\hline 1368.633 & 100 & $15.02(3) \mathrm{h}$ & & $15.6(4) \mathrm{h}$ & & $15.0(4) \mathrm{h}$ & \\
\hline 2754.028 & 99.94 & $15.05(3) \mathrm{h}$ & & $15.6(5) \mathrm{h}$ & & $14.9(5) \mathrm{h}$ & \\
\hline Na-24** & & 14.96(1) h & $2.72(16) E-29$ & 14.96(1) h & 4.83(40)E-29 & 14.96(1) h & 9.47(74)E-29 \\
\hline 1368.633 & 100 & $15.02(3) \mathrm{h}$ & & $15.6(4) \mathrm{h}$ & & $15.0(4) \mathrm{h}$ & \\
\hline 2754.028 & 99.94 & $15.05(3) \mathrm{h}$ & & $15.6(5) \mathrm{h}$ & & $14.9(5) \mathrm{h}$ & \\
\hline Br-82 & & $35.30(2) \mathrm{h}$ & 5.09(13)E-29 & $35.30(2) h$ & 6.37(12)E-28 & $35.30(2) h$ & 1.11(4)E-27 \\
\hline 554.348 & 70.8 & $1.08(5) \mathrm{d}^{*}$ & $6.90(77) \mathrm{E}-29$ & $1.45(5) \mathrm{d}$ & $6.82(20) \mathrm{E}-28$ & $1.25(5) \mathrm{d}$ & $1.17(10) \mathrm{E}-27$ \\
\hline 619.106 & 43.4 & $1.42(3) \mathrm{d}$ & $4.70(12) \mathrm{E}-29$ & $1.58(6) \mathrm{d}$ & $5.94(22) \mathrm{E}-28$ & $1.33(5) \mathrm{d}$ & $1.02(6) \mathrm{E}-27$ \\
\hline 698.374 & 28.5 & $1.44(4) \mathrm{d}$ & $4.64(14) \mathrm{E}-29$ & $1.68(7) \mathrm{d}$ & $6.06(30) \mathrm{E}-28$ & $1.74(20) \mathrm{d}$ & $1.35(8) \mathrm{E}-27$ \\
\hline 776.517 & 83.5 & $1.45(2) \mathrm{d}$ & $5.02(9) \mathrm{E}-29$ & $1.56(6) \mathrm{d}$ & $6.02(21) \mathrm{E}-28$ & $1.39(4) \mathrm{d}$ & $1.09(5) \mathrm{E}-27$ \\
\hline 827.828 & 24 & $1.45(5) \mathrm{d}$ & $5.43(17) \mathrm{E}-29$ & $1.53(7) \mathrm{d}$ & $6.30(24) \mathrm{E}-28$ & $1.41(2) \mathrm{d}$ & $0.99(10) \mathrm{E}-27$ \\
\hline 1044.002 & 27.2 & $1.48(5) \mathrm{d}$ & $5.31(18) \mathrm{E}-29$ & $1.52(6) \mathrm{d}$ & $6.55(25) \mathrm{E}-28$ & $1.7(3) \mathrm{d}$ & $1.13(11) \mathrm{E}-27$ \\
\hline 1317.473 & 26.5 & $1.54(5) \mathrm{d}$ & 5.61(17)E-29 & $1.61(7) \mathrm{d}$ & $6.59(28) \mathrm{E}-28$ & $1.32(11) \mathrm{d}$ & $1.15(9) \mathrm{E}-27$ \\
\hline 1474.880 & 16.3 & $1.45(6) \mathrm{d}$ & $5.39(20) \mathrm{E}-29$ & $1.58(6) \mathrm{d}$ & $6.56(26) \mathrm{E}-28$ & $1.36(12) \mathrm{d}$ & $1.11(8) \mathrm{E}-27$ \\
\hline I-123 & & 13.27(8) h & & 13.27(8) h & & & \\
\hline 158.970 & 83 & $12.3(6) \mathrm{h}$ & $1.26(16) E-29$ & $10(3) \mathrm{h}$ & $3.30(51) E-29$ & & \\
\hline I-124 & & $4.18(2) d$ & 2.42(15)E-29 & $4.18(2) d$ & & $4.18(2) d$ & \\
\hline
\end{tabular}




\begin{tabular}{|c|c|c|c|c|c|c|c|}
\hline 602.729 & 63 & $3.9(7) \mathrm{d}$ & $2.32(16) \mathrm{E}-29$ & $5.9(13) \mathrm{d}$ & $6.37(81)$ E-29 & $5.0(17) \mathrm{d}$ & $9.1(17)$ E-29 \\
\hline 1690.983 & 10.9 & $4.1(19) \mathrm{d}$ & 2.81(33)E-29 & & & & \\
\hline I-126 & & 13.11(5) d & $6.42(28) E-29$ & $13.11(5) d$ & 2.19(9)E-28 & 13.11(5) d & 3.92(20)E-28 \\
\hline 388.633 & 34.1 & $3.8(12) \mathrm{y}$ & $6.66(27) \mathrm{E}-29$ & $27(12) d$ & $2.23(10) \mathrm{E}-28$ & & $4.13(25) \mathrm{E}-28$ \\
\hline 666.331 & 33.1 & $4.4(19) \mathrm{y}$ & $6.05(30) \mathrm{E}-29$ & $26(12) d$ & $2.14(10) \mathrm{E}-28$ & & $3.68(28) \mathrm{E}-28$ \\
\hline 753.819 & 4.2 & $17(7) \mathrm{d}$ & $4.54(56) \mathrm{E}-29$ & & & & \\
\hline I-130 & & $12.36(3) \mathrm{h}$ & 4.59(10)E-27 & $12.36(3) \mathrm{h}$ & $1.07(2) E-26$ & $12.36(3) h$ & $1.84(4) E-26$ \\
\hline 418.010 & 34.2 & $12.47(2) \mathrm{h}$ & $4.15(7) \mathrm{E}-27$ & $12.8(4) \mathrm{h}$ & $1.01(4) \mathrm{E}-26$ & $12.27(29) \mathrm{h}$ & $1.76(6) \mathrm{E}-26$ \\
\hline 457.720 & 0.2 & $10.1(18) \mathrm{h}$ & $3.98(39) \mathrm{E}-27$ & & & & \\
\hline 536.090 & 99 & $12.44(2) \mathrm{h}$ & $4.48(7) \mathrm{E}-27$ & $12.8(4) \mathrm{h}$ & $1.05(4) \mathrm{E}-26$ & $12.2(4) \mathrm{h}$ & $1.81(6) \mathrm{E}-26$ \\
\hline 539.100 & 1.4 & $12.49(17) \mathrm{h}$ & $4.48(11) \mathrm{E}-27$ & $12.43(27) \mathrm{h}$ & $1.12(3) \mathrm{E}-26$ & $13.2(9) \mathrm{h}$ & $1.93(13) \mathrm{E}-26$ \\
\hline 553.900 & 0.7 & $1.08(5) \mathrm{d}^{*}$ & $8.31(8) \mathrm{E}-27 *$ & & & & \\
\hline 586.050 & 1.7 & $12.51(12) \mathrm{h}$ & $4.34(10) \mathrm{E}-27$ & $12.9(5) \mathrm{h}$ & $1.03(4) \mathrm{E}-26$ & $12.5(7) \mathrm{h}$ & $1.70(8) \mathrm{E}-26$ \\
\hline 603.500 & 0.6 & $13.8(5) \mathrm{h}$ & $5.35(20) \mathrm{E}-27$ & $13.4(18) \mathrm{h}$ & $1.13(9) \mathrm{E}-26$ & $2.37(15) \mathrm{d}^{*}$ & $2.12(22) \mathrm{E}-26$ \\
\hline 668.540 & 96 & $12.45(2) \mathrm{h}$ & $4.48(7) \mathrm{E}-27$ & $12.9(4) \mathrm{h}$ & $1.05(4) \mathrm{E}-26$ & $12.3(5) \mathrm{h}$ & $1.79(6) \mathrm{E}-26$ \\
\hline 685.990 & 1.1 & $12.53(16) \mathrm{h}$ & $4.33(10) \mathrm{E}-27$ & $13.1(5) \mathrm{h}$ & $0.98(4) \mathrm{E}-26$ & $12.9(18) \mathrm{h}$ & $1.91(13) \mathrm{E}-26$ \\
\hline 739.480 & 82 & $12.42(2) \mathrm{h}$ & 4.43(7)E-27 & $12.9(4) \mathrm{h}$ & $1.06(4) \mathrm{E}-26$ & $12.1(4) \mathrm{h}$ & $1.80(6) \mathrm{E}-26$ \\
\hline 800.230 & 0.1 & $11.6(19) \mathrm{h}$ & $4.17(43) \mathrm{E}-27$ & & & & \\
\hline 808.290 & 0.2 & $13.0(6) \mathrm{h}$ & $4.22(20) \mathrm{E}-27$ & 14.9(20) h & $0.92(8) \mathrm{E}-26$ & & \\
\hline 877.350 & 0.2 & $11.5(10) \mathrm{h}$ & $4.93(36) \mathrm{E}-27$ & & & & \\
\hline 967.020 & 0.9 & $12.47(24) \mathrm{h}$ & $4.51(11) \mathrm{E}-27$ & $13.3(8) \mathrm{h}$ & $1.08(5) \mathrm{E}-26$ & & $2.21(24) \mathrm{E}-26$ \\
\hline 1096.480 & 0.5 & $1.5(5) \mathrm{d}$ & $4.80(9) \mathrm{E}-27$ & $13.7(11) \mathrm{h}$ & $1.21(7) \mathrm{E}-26$ & & $2.34(35) \mathrm{E}-26$ \\
\hline 1122.150 & 0.2 & $12.7(10) \mathrm{h}$ & $5.60(30) \mathrm{E}-27$ & $16.4(25) \mathrm{h}$ & $1.44(19) \mathrm{E}-26$ & & \\
\hline 1157.470 & 11.3 & $12.37(4) \mathrm{h}$ & $5.31(10) \mathrm{E}-27$ & $13.1(5) \mathrm{h}$ & $1.11(4) \mathrm{E}-26$ & $11.8(4) h$ & $1.97(6) \mathrm{E}-26$ \\
\hline 1222.560 & 0.2 & $12.5(8) \mathrm{h}$ & $5.54(27) \mathrm{E}-27$ & & & & \\
\hline 1272.120 & 0.7 & $12.65(24) \mathrm{h}$ & $5.67(13) \mathrm{E}-27$ & $13.6(7) \mathrm{h}$ & $1.24(7) \mathrm{E}-26$ & $10(4) \mathrm{h}$ & $2.45(30) \mathrm{E}-26$ \\
\hline 1403.900 & 0.3 & $12.3(3) \mathrm{h}$ & $5.08(16) \mathrm{E}-27$ & $14.1(20) \mathrm{h}$ & $1.08(12) \mathrm{E}-26$ & & \\
\hline
\end{tabular}




\section{CONCLUSIONS}

Is interesting to compare the results for ${ }^{232} \mathrm{Th}$ of this work with the results of other experiments. In [33] on setup «GAMMA-2»: $\mathrm{Pb} \operatorname{target}(\mathrm{d}=8 \mathrm{~cm}, 1=20 \mathrm{~cm})$, paraffin moderator $\left(6 \mathrm{~cm}\right.$ thick). ${ }^{232} \mathrm{Th}$ sample located on the end surface of the target. Installation was irradiated by protons with an energy of $1 \mathrm{GeV}$. The reaction rate $(\mathrm{n}, \gamma) \mathrm{R}=9.80(6) \mathrm{E}-27$, almost equal to the results of this work at a deuteron energy $2 \mathrm{GeV}$. In [9] on the setup "Energy plus Transmutation": overall length $48 \mathrm{~cm}$ (4 sections), the length of $\mathrm{Pb}$ target in four sections of $45.6 \mathrm{~cm}$ and a diameter of 8.4 $\mathrm{cm}$ target surrounded by uranium blanket thickness of two elements (see present work) and irradiated with deuterons $1.6 \mathrm{GeV}$. Samples located on the surface of the blanket. The reaction rate $(\mathrm{n}, \gamma) \mathrm{R}=3.03(10) \mathrm{E}-26$ and fission $(\mathrm{n}, \mathrm{f}) \mathrm{R}=5.89(60) \mathrm{E}-27$ - 3 and 10 times higher than results of this work at $2 \mathrm{GeV}$. Also interesting comparison with the work [17], made at a deuteron energy of $2.33 \mathrm{GeV}$ at the setup "GAMMA-3": a lead target $(\mathrm{d}=8 \mathrm{~cm}, \mathrm{l}=60 \mathrm{~cm})$ in the carbon moderator with large size, channels for samples at different distances from the center of the target. Results for $\mathrm{R}-(\mathrm{n}, \gamma)$ in a yield of $233 \mathrm{~Pa}$ (will eventually $233 \mathrm{U}$ ) is $30-40$ times higher and fission (n, f) - 20 - 30 times higher than present work for the nearest window on carbon ( $24 \mathrm{~cm}$ from the axis target). This is due to the high ability of carbon to slowing of spallation neutrons coming from the target.

Ratio of the weight of produced ${ }^{233} \mathrm{U}$ to ${ }^{232} \mathrm{Th}$ at $2 \mathrm{GeV}-2.90(17) \mathrm{E}-13$ at $4 \mathrm{GeV}$ $4.88(8) \mathrm{E}-13$ and at $8 \mathrm{GeV} 3.93(18) \mathrm{E}-13$. Ratio of the weight of produced ${ }^{130} \mathrm{I}$ to ${ }^{129} \mathrm{I}$ in the conditions of the present experiment at $2 \mathrm{GeV} 1.40(3) \mathrm{E}-13$ at $4 \mathrm{GeV} 2.94(6) \mathrm{E}-$ 13 and at $8 \mathrm{GeV} 1.69(4) \mathrm{E}-13$. If we calculate of transmutation for conditions: $10 \mathrm{~mA}$ and irradiate for 30 days. It is: $2 \mathrm{GeV}-0.082(2) \% ; 4 \mathrm{GeV}-0.190(4) \%$ and $8 \mathrm{GeV}$ $-0.330(7) \%$. These calculations allow us to estimate the transmutation at high currents and irradiation time - in the tens of percent. By Calc. 1 and experimental data $90(5) \%$ of the transmutation ${ }^{129} \mathrm{I}$ is $(\mathrm{n}, \gamma)$ reaction at all three deuteron energies $(2,4,8 \mathrm{GeV})$.

These results are interesting to compare with those obtained previously. The first work was done with ${ }^{129} \mathrm{I}$ in the direct beam of protons Phasotron JINR $\mathrm{E}_{\mathrm{p}}=660$ $\mathrm{MeV}$ and a current of $1.2 \mathrm{~mA}$ [34]. Samples with ${ }^{129} \mathrm{I}$ and ${ }^{127} \mathrm{I}$ were in direct proton beam. The paper presents number: 30 days and $10 \mathrm{~mA}$. However, during its obtaining were taken coefficient "2" - taking into account the formation of stable and long-lived isotopes, as well as registration of gamma rays with energies lower than $300 \mathrm{keV}$ (samples ${ }^{129} \mathrm{I}$ are measured with a filter: $\mathrm{Pb}-10 \mathrm{~mm}, \mathrm{Cd}-2 \mathrm{~mm}$ and $\mathrm{Cu}-$ $1 \mathrm{~mm})$. Therefore, comparisons should take a number (2-3)\%. It should be borne in 
mind that the experimental conditions: the direct beam of protons and not heavy target surrounded uranium, carbon or paraffin moderator - significantly different from the next. In experiments with beams of relativistic protons nuclotron JINR VBLHEP with energies: 1.0, 1.5 and $2.0 \mathrm{GeV}$ [10] - transmutation is respectively, $0.075,0.132$ and $0.153 \%$ at a current of $10 \mathrm{~mA}$ and 30 days of irradiation, which is close to the results of this work. In a similar study with deuterons $2.52 \mathrm{GeV}$ [8], a transmutation of $0.132 \%$. In [11] the results for protons with energy $2.0 \mathrm{GeV}$ transmutation is $0.13 \%$. In $[35,36]$ at the proton beam with the energy of $3.67 \mathrm{GeV}$ (Nuclotron JINR), lead target ( $\mathrm{d}=8 \mathrm{~cm}, 1=20 \mathrm{~cm}$, paraffin moderator) transmutation of ${ }^{129} \mathrm{I}$ was $0.9 \%$ (10 mA, 30 days). Grows of transmutation is associated from energy, obviously with increase the yield of spallation neutrons from the target (70 $\pm 20 \%)$ and including - thermal, making the main contribution to the reaction $(n, \gamma)$ more than $92 \%$.

\section{REFERENCES}

1. C.D. Bowman et al. // Nucl. Instr. Meth. A. 1992. V.320. P.336.

2. A.J. Janssen. Transmutation of fission products in reactors and Accelerator-Driven Systems, ECN-R-94-001. 2004.

3. C. Rubbia, J.A. Rubio et al. Conceptual design of a fast neutron operated high power energy amplifier CERN/AT/95-44 (ET).1995. See also C. Rubbia. High gain energy amplifier operated with fast neutrons. AIP Conference 346, Proceedings of International Conference on Accelerator-Driven Transmutation Technologies and Applications, Las Vegas (NV), US.1994.

4. Thorium Fuel Cycle - Potential Benefits and Challenges, IAEA-TECDOC-1450 (2005).

5. G.N. Kim et al. // Nucl. Instr. Meth. A. 2002. V.485. P.458.

6. http://pceet075.cern.ch/

7. http://www.sckcen.be/myrrha/

8. M.I. Krivopustov, A.V. Pavlyuk, A.I. Malakhov et al. About the first experiment on investigation of ${ }^{129} \mathrm{I},{ }^{237} \mathrm{~Np},{ }^{238} \mathrm{Pu}$ and ${ }^{239} \mathrm{Pu}$ transmutation at the Nuclotron $2.52 \mathrm{GeV}$ deuteron beam in neutron field generated in $\mathrm{U} / \mathrm{Pb}$ assembly "Energy plus Transmutation". Journal of Radioanalytical and Nuclear Chemistry, Vol. 279, No.2 (2009) 567-584 // Preprint JINR E1-2007-7. 2007. Dubna.

9. J. Adam, K. Katovsky, M. Majerle et al. Transmutation of Th and U with neutrons produced in $\mathrm{Pb}$ target and U-blanket system by relativistic deuterons. Eur. Phys. J. A 43. No2. pp. 159-173, 2010. // Preprint JINR, E15-2008-118. Dubna, 2008, 29 p.

10. J. Adam, K. Katovsky, A. Balabekyan et al. Transmutation of ${ }^{129} \mathrm{I},{ }^{237} \mathrm{~Np},{ }^{238} \mathrm{Pu},{ }^{239} \mathrm{Pu}$ and ${ }^{241} \mathrm{Am}$ Using Neutrons Prodyused in Target-Blanket System "Energy plus Transmutation" by relativistic Protons. Pramana Journal of Physics. Vol.68, N 2 (2007). PP. 201-212.

11. J. Adam, K. Katovsky A. R. Balabekyan et al. Transmutation of ${ }^{129} \mathrm{I},{ }^{237} \mathrm{~Np},{ }^{239} \mathrm{Pu}$ and ${ }^{241} \mathrm{Am}$ Using Neutrons Produced in Pb-Target with ${ }^{\text {nat }} \mathrm{U}$ Blanket System "Energy plus Transmutation" Bombarded by $2.0 \mathrm{GeV}$ Relativistic Protons. 
Proc.of the International Conference on Nuclear Data and technology. Seotember 26Oktoder 1,2004, Santa Fe, New Mexico, USA. N.Y.,2005. V.2. P 1560-1562.

12. http://sinq.web.psi.ch/

13. http://www.kek.jp/intra-e/index.html

14. http://lansce.lanl.gov/indexC.html

15. Коллаборация «Энергия и трансмутация радиоактивных отходов». Исследование пространственных распределений реакций деления и радиационного захвата нейтронов в массивной урановой мишени, облучаемой дейтронами с энергией 1-8 ГэВ (установка «Квинта»). Препринт ОИЯИ, Р1-2012-147, Дубна, 2012, 20 с.

16. N.L. Asquith, S.R. Hashemi-Nezhad, S. Tyutyunnikov, M. Kadykov, V. Chilap, J. Adam, W. Furman. Annals of Nuclear Energy. Vol. 63, 2014, P. 742-750

17. J.Adam et.al. A study of rates of $(n, f),(n, \gamma)$, and $(n, 2 n)$ reactions in ${ }^{\text {nat }} U$ and ${ }^{232} \mathrm{Th}$ produced by the neutron fluence in the graphite setup (GAMMA-3) irradiated by 2.33 GeV. Eur. Phys. J. A 47, 85-104 (2011). (Preprint JINR. E1-2011-52, Dubna 2011)

18. M. Majerle et al. Spallation experiment on thick lead target: analysis of experimental data with Monte Carlo codes. Preprint JINR, E15-2008-94. Dubna, 2008, 20 p.

19. J. Adam et al. Investigation of the formation of residual nuclei in rections induced by 660 $\mathrm{MeV}$ protons interacting with the radioactive ${ }^{237} \mathrm{~Np},{ }^{241} \mathrm{Am}$ and ${ }^{129} \mathrm{I}$ targets. Journal of Nuclear Science and Technology, Supplement 2, p. 272-275, 2002.

20. V. Henzl et al. Transmutation of ${ }^{129}$ I with high energy neutrons produced in spallation reactions induced by protons in massive target. Journal of Nuclear Science and Technology, Supplement 2, p. 1248-1251, 2002.

21. J. Frana. Program DEIMOS32 for gamma-ray spectra evaluation. J. Radioanal. Nucl. Chem. 2003, V. 257, 583.

22. И. Адам и др. Система программ и дополнения к методу активационного анализа для определения сечений ядерных реакций. Измерительная техника. №1, 2001, 57-61. //Препринт ОИЯИ, Р10-2000-28, Дубна, 2000, 22 с.

23. И. Адам и др. Исследование образования продуктов протон-ядерных реакций в мишени ${ }^{129}$ І при энергии протонов 660 МэВ. Письма в ЭЧАЯ. 2004. Т. 1, № 4(121). C. 53-64.

24. N.V. Mokhov, C.C. James. The MARS Code System User's Guide: Version 15. Fermi National Accelerator Laboratory. P.O. Box 500, Batavia, Illinois 60510, January, 2014.

25. S.G. Mashnik, et al., LANL Report LA-UR-08-2931, 2008.

26. D.P. Pelowitz, MCNPX User's Manual: Version 2.5.0, Technical Report, LA-CP- 050369, Los Alamos National Laboratory and University of California, April 2005.

27. A. Boudard, et al., Physical Review C 66 (2002) 044615.

28. A.R. Junghans, et al., Nuclear Physics A 629 (1998) 635.

29. A. J. Koning, TALYS User Manual: Version 1.4, Technical Report, NL-1755 ZG, Petten, The Netherlands, December 2011.

30. TENDL-2009, Nuclear data library.

31. JEFF3.1.2, Nuclear data library.

32. O.A. Shcherbakov, A.Y. Donets, A.V. Evdokimov, A.V. Fomichev, T. Fukahori, A. Hasegawa, A.B. Laptev, V.M. Maslov, G.A. Petrov, Y.V. Tuboltsev, A.S. Vorobiev, Journal of Nuclear Science and Technology Supplement 2 (1) (2002) 230.

33. J. Adam, A.R. Balabekyan, V.S. Barashenkov et al. Spallation neutron spectrum on a massive lead/paraffin target irradiated with $1 \mathrm{GeV}$ protons. Eur. Phys. J. A 23, 61-68 (2005). // Preprint JINR E1-2004-16. 13 pp. Dubna. 2004. 
34. И. Адам, А. Балабекян, В.С. Барашенков и др. Исследование образования продуктов протон-ядерных реакций в мишени ${ }^{129} \mathrm{I}$ при энергии протонов 660 МэВ. Труды 51 междунар. сов. по ядерной спектроскопии и структ. атомного ядра. Саратов, Россия, 2001, с.201. // Письма в ЭЧАЯ. 2004. т.1, № 4 (121).С.53-64.

35. M.I. Krivopustov, J. Adam, V. Bradnova et al. First Experiment on Transmutation Studies of Iodine - 129 and Neptunium -237 Using Relativistic Protons of 3.7 GeV. Preprint JINR, E1-97-59 (1997). Dubna.// J. of Radioanalytical and Nuclear Chemistry. Vol.222 (1997).P.267

36. J. S. Wan, M. Ochs, R. Brandt et al. Transmutation of radioactive waste by means of relativistic heavy ions. Kerntechnik 63 (1998) 4. // Comunication JINR, E1-99-1, Dubna. 1999. 